Groups Geom. Dyn. 7 (2013), 883-910

DOI $10.4171 / \mathrm{GGD} / 209$
Groups, Geometry, and Dynamics

(C) European Mathematical Society

\title{
Free groups of interval exchange transformations are rare
}

\author{
François Dahmani, Koji Fujiwara and Vincent Guirardel
}

\begin{abstract}
We study the group IET of all interval exchange transformations. Our first main result is that the group generated by a generic pairs of elements of IET is not free (assuming a suitable irreducibility condition on the underlying permutation). Then we prove that any connected Lie group isomorphic to a subgroup of IET is abelian.

Additionally, we show that IET contains no infinite Kazhdan group. We also prove residual finiteness of finitely presented subgroups of IET and give an example of a two-generated subgroup of IET of exponential growth that contains an isomorphic copy of every finite group and which is therefore not linear.
\end{abstract}

Mathematics Subject Classification (2010). 37E05, 20E07, 37A15, 20 F38.

Keywords. Groups of diffeomorphisms, interval exchange transformation, Lie groups, free groups.

Consider an interval. Break it into finitely many subintervals. Rearrange these pieces in the interval (preserving the orientation) to obtain a bijection. This is an interval exchange transformation. More precisely, an interval exchange transformation of $[0,1)$ is a left-continuous bijection, with finitely many discontinuity points, which is piecewise a translation. The set IET of all interval exchange transformations is a group under composition.

Interval exchange transformations have been widely studied for themselves, in the point of view of the dynamical system they individually generate (for an introduction see [5], Chap. 14.5). Considerably less is known about the global structure of the group IET.

Basic test questions concern the possible subgroups of IET. For instance, the answer to the following question raised by Katok is still unknown.

Question (Katok). Does IET contain a non-abelian free group?

Our first result concerns the subgroups of IET isomorphic to a connected Lie group, a question raised by Franks. Using rotations with disjoint support, one can easily realize the $n$-dimensional torus $\mathbb{T}^{n}$ as a subgroup of IET for any $n$ (therefore, one can also realize $\mathbb{R}^{n}$ and any connected abelian Lie group). We prove 
Theorem 4.4. A connected Lie group embeds as a subgroup of IET if and only if it is isomorphic to $\mathbb{T}^{n} \times \mathbb{R}^{m}$.

The embedding in the statement is purely algebraic, it is not assumed to have any continuity property. A similar result was recently independently obtained by Novak [10], [11]. It is also known that there is no finitely generated subgroup of IET that has a distorted element for a word metric [9]. This excludes most nilpotent groups but does not exclude $\mathrm{SO}_{3}(\mathbb{R})$.

Because translations commute, the orbit of any point under a finitely generated subgroup of IET has polynomial growth. In particular, a possible free subgroup of IET cannot be produced by controling only the action on a single point, hence this kind of ping-pong will fail. Following an observation by Witte Morris, we show that this implies that the image in IET of any finitely generated Kazhdan group is finite.

But polynomial growth of orbits does not imply polynomial growth of the group, and we produce an example of subgroup of IET containing a free semigroup.

Theorem 8.1. There is a 2-generated subgroup $G$ of IET such that

- $G$ contains a free semigroup,

- $G$ contains an isomorphic copy of every finite group.

In particular, $G$ is not linear.

In the context of connected Lie groups, the existence of a free subgroup of a connected lie group $G$ implies that a generic subgroup is free. Here a generic subset is a subset containing a countable intersection of dense open subsets. Indeed, for each reduced word in two letters and their inverses, the subset $V_{w} \subset G \times G$ of pairs $(a, b) \in G \times G$ with $w(a, b)=1$ is a proper analytic subset of $G \times G$ (it is not $G \times G$ because there exists a free subgroup). Since $G$ is connected, $V_{w}$ has empty interior. Baire's theorem then implies that a generic subset of $G \times G$ consists of generators of free non-abelian subgroups.

There is a natural topology on IET that allows to talk about genericity. Given a permutation $\sigma$ of $\{1, \ldots, n\}$, the set $\mathrm{IET}_{\sigma}$ of all interval exchange transformations with $n-1$ points of discontinuity and with underlying permutation $\sigma$ is naturally in bijection with the $(n-1)$-dimensional open simplex. This gives a natural topology

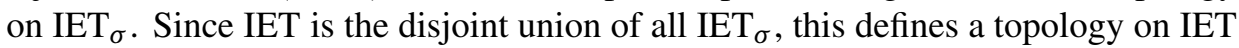
by declaring all IET $\sigma$ open in IET. For this topology, a generic subset of IET is a subset that intersects each $\mathrm{IET}_{\sigma}$ in a generic subset. Note however that this does not make IET a topological group as the group law is discontinuous.

The genericity argument used above for a connected Lie group does not work in IET. Indeed, the subset $V_{w} \subset$ IET $\times$ IET is not an analytic subset but looks like a union of polyhedra and can have non-empty interior. In fact, we prove that the situation is opposite to that of non-solvable connected Lie groups: if one restricts to admissible permutations (as defined below), the group generated by a generic pair 
of elements is not free. This is a beginning of an explanation of the fact that free subgroups of IET seem difficult to find.

Theorem 5.2. Let $\mathrm{IET}_{a}$ be the set of interval exchange transformations whose underlying permutation is admissible.

Then there is a dense open subset of IET $\times \mathrm{IET}_{a}$ such that the group generated by any pair in this subset is not free.

In this statement a permutation of $\{1, \ldots, n\}$ is admissible if there is no $m$ such that $\sigma(m)=m$ and $\{1, \ldots, m-1\} \sigma$-invariant. The subset IET $a \subset$ IET is the (open) set of all transformations in IET with admissible underlying permutation.

Finally we prove a simple additional result for subgroups of IET that excludes the existence of Thompson groups in IET.

Theorem 7.1. Any finitely presented subgroup of IET is residually finite.

More generally, any finitely generated subgroup is a limit of finite groups in the space of marked groups. In particular, IET is sofic.

The methods of this paper are rather elementary, and the paper is almost selfcontained.

Our first observation, similar to that in [9], allows to establish that elements admitting roots of arbitrarily high order are conjugated to so-called virtual multirotations. Denoting by $\operatorname{Disc}(h)$ the number of discontinuity points of an IET $h$, define its growth rate $\|h\|=\lim _{n \rightarrow \infty} \frac{1}{n} \operatorname{Disc}\left(h^{n}\right)$. Obvious properties are that $\|h\|$ is a conjugacy invariant, $\|h\| \leq \operatorname{Disc}(h)$, and $\left\|h^{k}\right\|=k\|h\|$ for $k \geq 0$. We prove that if one allows to change $[0,1)$ to a more complicated domain (a union of circles and intervals), then there any IET has a conjugate that is optimal with respect to the growth of its number of discontinuity points:

Proposition 2.4. For any $h \in \mathrm{IET}$, there exists another domain $\mathscr{D}_{m}$ and an IET $h_{m}$ conjugate to $h$ such that $\operatorname{Disc}\left(h_{m}\right)=\left\|h_{m}\right\|=\|h\|$.

This implies that $\|h\|$ is an integer. In particular, any divisible element satisfies $\|h\|=0$ and is therefore conjugate to a continuous IET: a virtual multi-rotation. This can be used to compute centralizers as in [9] (see Corollary 2.7).

We then argue that no non-abelian free group contains a multi-rotation. In fact, for every multi-rotation $S$ and for every interval exchange transformation $T$, we can play a game of computing commutators to obtain an interval exchange transformation with small support in the generated subgroup $\langle S, T\rangle$. Then one can conjugate this element to an element with disjoint support. This exhibits a commutation relation that prevents $\langle S, T\rangle$ from being free non-abelian.

Since connected Lie groups have mostly elements admitting roots of arbitrarily high order, if they are realized as subgroup of IET they cannot contain any nonabelian free group, therefore they must be solvable. This already rules out groups 
like $\mathrm{SO}_{3}(\mathbb{R})$. For solvable connected Lie groups, we investigate the metabelian case to conclude that they must be abelian.

In order to address our genericity theorem, we argue that interval exchange transformations whose subdivision points are well approximated by rational numbers almost behave like those ones with rational subdivision points. The latter are of finite order and this allows again using commutators to obtain elements with small supports. The next step again is to find elements with disjoint supports to deduce commutation relations. This is where we need the assumption that the underlying permutation of at least one of the elements is admissible.

The paper is organized as follows. Section 1 is mainly devoted to definitions concerning IETs. Section 2 constructs our minimal model for discontinuity points. Section 3 shows that multi-rotations cannot be contained in a free group. Section 4 classifies which Lie groups are subgroups of IET. Section 5 shows that generically, pairs of elements do not generate a free group. Sections 6 and 7 show that infinite Kazhdan groups and Thompson groups are not subgroups of IET. Section 8 explains the example of a 2-generated subgroup of exponential growth containing all finite groups.

We would like to thank A. Katok, D. Calegari, E. Breuillard and D. Witte-Morris for related discussions. We also thank the referee for useful suggestions. The second author is in part supported by Grant-in-Aid for Scientific Research (No. 19340013). He also acknowledges the hospitality of Institut de Mathématiques de Toulouse.

\section{Preliminaries}

1.1. Interval exchange transformations. A domain $\mathscr{D}$ is a disjoint finite union of circles of the form $\mathbb{R} / l \mathbb{Z}$ and of semi-open bounded intervals $[\alpha, \beta)$. Each component of $\mathscr{D}$ carries a natural metric and an orientation.

An interval exchange transformation $h: \mathscr{D} \rightarrow \mathscr{D}^{\prime}$ between two domains $\mathscr{D}, \mathscr{D}^{\prime}$ is a bijective map $h: \mathscr{D} \rightarrow \mathscr{D}^{\prime}$ which is piecewise isometric, orientation-preserving, continuous on the right, and with only finitely many discontinuity points. We denote by $\operatorname{IET}\left(\mathscr{D}, \mathscr{D}^{\prime}\right)$ the set of interval exchange transformation from $\mathscr{D}$ to $\mathscr{D}^{\prime}$. Interval exchange transformations may be composed, and the set IET $(\mathscr{D}, \mathscr{D})$ is a group which we denote by IET $(\mathscr{D})$ (in fact, the set of all interval exchange transformations has a structure of a groupoid, but we will not make use of this terminology). We also define $\operatorname{IET}=\operatorname{IET}([0,1))$

The continuity intervals of $h$ are the maximal connected subsets of $\mathscr{D}$ on which $h$ is continuous. These are finitely many semi-open intervals or circles that partition $\mathscr{D}$.

We say that $g \in \operatorname{IET}(\mathscr{D})$ and $g^{\prime} \in \operatorname{IET}\left(\mathscr{D}^{\prime}\right)$ are conjugate if there exists $h \in$ $\operatorname{IET}\left(\mathscr{D}, \mathscr{D}^{\prime}\right)$ with $g^{\prime}=h g h^{-1}$. There exists an interval exchange transformation $h$ between $\mathscr{D}$ and $\mathscr{D}^{\prime}$ if and only if $\mathscr{D}, \mathscr{D}^{\prime}$ have the same total length. In this case conjugation by $h$ induces an isomorphism between $\operatorname{IET}(\mathscr{D})$ and $\operatorname{IET}\left(\mathscr{D}^{\prime}\right)$. Note that 
even if $\mathscr{D}$ and $\mathscr{D}^{\prime}$ do not have the same total length, conjugation by a homothety shows that $\operatorname{IET}(\mathscr{D}) \simeq \operatorname{IET}\left(\mathscr{D}^{\prime}\right)$.

A subdomain of $\mathscr{D}$ is a subset of $\mathscr{D}$ which is a finite union of semi-open intervals and circles. Interval exchange transformations map subdomains to subdomains. We define the support of $g \in \operatorname{IET}(\mathscr{D})$ as $\operatorname{supp}(g)=\{x \in \mathscr{D} \mid g(x) \neq x\}$ (and not its closure). It is a subdomain of $\mathscr{D}$ and is natural under conjugation: $\operatorname{supp}\left(h g h^{-1}\right)=$ $h(\operatorname{supp}(g))$.

1.2. Multi-rotations. If $\mathcal{D}$ is a circle, a continuous interval exchange is a rotation. We define a multi-rotation as an interval exchange transformation of some domain $\mathcal{D}$, which preserves each component on $\mathcal{D}$ and which restricts to a rotation on each circle of $\mathscr{D}$ and to the identity on each segment. More generally, a virtual multirotation is any continuous element of IET(D). Clearly, multi-rotations are virtual multi-rotations, and any virtual multi-rotation has a power which is a multi-rotation.

Say that a multi-rotation $R$ is irrational if $R$ has infinite order, and the restriction of $R$ to each circle is either the identity or has infinite order (i.e., is an irrational rotation). Note that any multi-rotation of infinite order has a power which is an irrational multi-rotation. If $R$ is conjugate to an irrational multi-rotation, then $R$ defines a free action of $\mathbb{Z}$ on the support of $R$.

The following is probably well known.

Lemma 1.1. Let $R$ be an irrational rotation on a circle $C$.

(1) If $R^{\prime}$ commutes with $R$, then $R^{\prime}$ is a rotation.

(2) If $S \in \operatorname{IET}(\mathcal{C})$ is such that $S R S^{-1}$ commutes with $R$. then $S$ is itself a rotation, and in particular, $S$ commutes with $R$.

Proof. For $R^{\prime} \in$ IET, denote by $\Delta\left(R^{\prime}\right)$ its set of discontinuity points. Since $R, R^{-1}$ are continuous, $\Delta\left(R R^{\prime} R^{-1}\right)=R\left(\Delta\left(R^{\prime}\right)\right)$. In particular, if $R^{\prime}$ commutes with $R$, $\Delta\left(R^{\prime}\right)$ is $R$-invariant. Being finite, $\Delta\left(R^{\prime}\right)$ has to be empty and $R^{\prime}$ is a rotation. This proves the first assertion.

If $R^{\prime}=S R S^{-1}$ commutes with $R$, then $R^{\prime}$ is a rotation. By continuity of $R$ and $R^{\prime}$, the relation $R^{\prime} S=S R$ yields $\Delta(S)=R^{-1} \Delta(S)$, which implies that $\Delta(S)=\varnothing$ as above.

\subsection{Irrational circles}

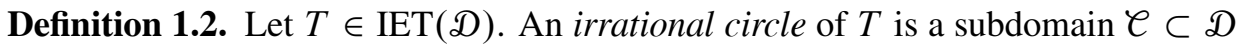
(not necessarily homeomorphic to a circle, or even connected) which is $T$-invariant and such that $\left.T\right|_{e}$ is conjugate to an irrational rotation on a circle.

For example, given $0<\tau<l<1$, consider the transformation $h:[0,1) \rightarrow[0,1)$ defined as the translation of length $\tau$ on $[0, l-\tau)$, as the translation of length $\tau-l$ 
on $[l-\tau, l)$, and as the identity on $[l, 1)$. Then $[0, l)$ is an irrational circle of $h$ if and only if $\tau / l \notin \mathbb{Q}$.

Of course, $T$ is conjugate to an irrational multi-rotation if and only if its support is a disjoint union of irrational circles.

It follows from the definition that if $g^{\prime}=h g h^{-1}$ and if $\mathcal{C}$ is an irrational circle of $g$, then $h(\mathcal{C})$ is an irrational circle of $g^{\prime}$.

Lemma 1.3. For any $T \in \operatorname{IET}(D)$, two irrational circles of $T$ either are disjoint or coincide.

More generally, if a subdomain $\mathscr{D}_{0} \subset \mathbb{D}$ intersects some irrational circle $\mathcal{C}$ and if $T\left(\mathscr{D}_{0}\right)=\mathscr{D}_{0}$, then $\mathscr{D}_{0} \supset \mathcal{C}$.

Proof. The first statement follows from the second. Assume that there is some point $x \in \mathscr{D}_{0} \cap \mathcal{C}$. Then $\mathscr{D}_{0} \cap \mathcal{C}$ contains a small interval $I=[x, x+\varepsilon)$. Since $\mathscr{D}_{0}$ is $T$-invariant, $T^{i}(I) \subset \mathscr{D}_{0}$ for all $i \in \mathbb{Z}$. Since $\left.T\right|_{\mathcal{C}}$ is conjugate to an irrational rotation, there exists $i$ such that $\mathcal{C}=I \cup T(I) \cup \cdots \cup T^{i}(I)$, and the lemma follows.

Lemma 1.4. Let $S, T \in \mathrm{IET}(D)$ be conjugate to irrational multi-rotations.

If $S, T$ commute, then for each irrational circle $\mathcal{C}$ of $S$, either $\mathcal{C}$ does not intersect $\operatorname{supp}(T)$, or $\mathcal{C}$ is an irrational circle of $T$.

Proof. Since $S$ and $T$ commute, $T(\mathcal{C})$ is an irrational circle of $S$. Since $S$ has only finitely many irrational circles, there exists $k \geq 1$ such that $T^{k}(\mathcal{C})=\mathscr{C}$. Assume that $\mathcal{C}$ intersects $\operatorname{supp}(T)$, i.e., that it intersects some irrational circle $\mathcal{C}_{T}$ of $T$. By Lemma 1.3 (applied to the transformation $T^{k}$ and to $D_{0}=\ell$ ), $\ell_{T} \subset \mathcal{C}$. By

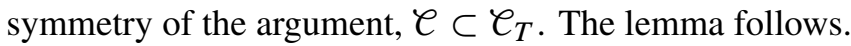

Lemma 1.5. Let $S$ and $T$ be conjugate to irrational multi-rotations. If $S^{n} T S^{-n}$ commutes with $T$ for all $n$, then $S$ commutes with $T$.

Proof. We prove that for each irrational circle $\ell_{T}$ of $T, S$ preserves $\ell_{T}$ and $S \mid e_{T}$ commutes with $T \mid e_{T}$. The lemma follows immediately.

We can assume that $\ell_{T}$ intersects the support of $S$, hence some irrational circle $\mathcal{C}_{S}$ of $S$. Let $I=[x, x+\varepsilon)$ be a segment in $\mathcal{C}_{S} \cap \mathcal{C}_{T}$. There is $n \geq 1$ such that $S^{n}(I) \cap I \neq \emptyset$, so $S^{n}\left(\mathcal{C}_{T}\right)$ intersects $\mathcal{C}_{T}$. Since $S^{n}\left(\mathcal{C}_{T}\right)$ is an irrational circle of $S^{n} T S^{-n}$ which commutes with $T, S^{n}\left(\mathcal{C}_{T}\right)=\mathcal{C}_{T}$ by Lemma 1.4.

Applying Lemma 1.3 to the $S^{n}$-invariant subdomain $\mathcal{C}_{T}$, we get $\mathcal{C}_{T} \supset \mathcal{C}_{S}$. Since this holds for all irrational circle $\ell_{S}$ which intersect $\ell_{T}, \ell_{T}$ is $S$-invariant. Since $T \mid e_{T}$ is conjugate to an irrational rotation, we can apply Lemma 1.1 to the restrictions of $T$ and $S$ to $\mathcal{C}_{T}$, and get that $S \mid e_{T}$ commutes with $T \mid e_{T}$. 


\section{Points of discontinuity}

\subsection{Definitions}

Definition 2.1. Given $h: \mathscr{D} \rightarrow \mathscr{D}^{\prime}$ an interval exchange map, we denote by $\Delta(h)$ the finite set of points in $\mathscr{D}$ where $h$ is discontinuous, and by $\operatorname{Disc}(h)=\# \Delta(h)$ its cardinal.

We define the interior $\stackrel{D}{D}$ of $\mathscr{D}$ as the set of points $x$ having a neighbourhood isometric to an open interval $(-\varepsilon, \varepsilon)$. We note that $h$ being continuous on the right, it is automatically continuous at points in $\mathscr{D} \backslash \grave{D}$. Conversely, if $h(x) \in \mathscr{D} \backslash \grave{D}$ and $x \in \stackrel{\circ}{\mathrm{D}}$, then $h$ is not continuous at $x$.

Lemma 2.2. Consider $h \in \operatorname{IET}\left(\mathscr{D}, \mathscr{D}^{\prime}\right), h^{\prime} \in \operatorname{IET}\left(\mathscr{D}^{\prime}, \mathscr{D}^{\prime \prime}\right)$.

Then $\operatorname{Disc}\left(h^{\prime} \circ h\right) \leq \operatorname{Disc}(h)+\operatorname{Disc}\left(h^{\prime}\right)$.

Proof. For any $x \in \Delta\left(h^{\prime} \circ h\right)$, then either $x \in \Delta(h)$ or $h(x) \in \Delta\left(h^{\prime}\right)$ as the composition of continuous maps is continuous. Thus $\Delta\left(h^{\prime} \circ h\right) \subset \Delta(h) \cup h^{-1} \Delta\left(h^{\prime}\right)$. Since $h$ is a bijection, the lemma follows.

Lemma 2.3. Given $h \in \operatorname{IET}(D)$, we can define

$$
\|h\|=\inf \left\{\frac{1}{n} \operatorname{Disc}\left(h^{n}\right) \mid n \in \mathbb{N}\right\}=\lim _{n \rightarrow \infty} \frac{1}{n} \operatorname{Disc}\left(h^{n}\right) .
$$

It satisfies $\left\|h^{k}\right\|=k\|h\|$ for all $k \in \mathbb{N}$ and if $h, h^{\prime}$ are conjugate, then $\|h\|=\left\|h^{\prime}\right\|$.

Proof. The limit exists and coincides with the infimum by subadditivity (Lemma 2.2). For any $h \in \operatorname{IET}(\mathcal{D}),\left\|h^{k}\right\|=\lim _{n \rightarrow \infty} \frac{1}{n} \operatorname{Disc}\left(h^{k n}\right)=k \lim _{n \rightarrow \infty} \frac{1}{k n} \operatorname{Disc}\left(h^{k n}\right)=$ $k\|h\|$. If $h^{\prime}=g h g^{-1}$, then $h^{\prime n}=g h^{n} g^{-1}$, $\operatorname{so} \operatorname{Disc}\left(h^{\prime n}\right) \leq \operatorname{Disc}(g)+\operatorname{Disc}\left(h^{n}\right)+$ $\operatorname{Disc}\left(g^{-1}\right)$. Passing to the limit, we get $\left\|h^{\prime}\right\| \leq\|h\|$. Since the symmetric inequality holds, the lemma follows.

\subsection{Minimal model for discontinuity points and applications}

Proposition 2.4. Let $h \in \operatorname{IET}(\mathcal{D})$. Then there exists another domain $\mathscr{D}_{m}$ and $h_{m} \in \operatorname{IET}\left(\mathscr{D}_{m}\right)$ conjugate to $h$, such that $\operatorname{Disc}\left(h_{m}^{n}\right)=n \operatorname{Disc}\left(h_{m}\right)$ for all $n \in \mathbb{N}$.

This conjugate $h_{m}$ has the minimal number of discontinuity points among all conjugates of $h$ as shows the third assertion of the following corollary.

Corollary 2.5. Consider any $h \in \operatorname{IET}(\mathcal{D})$. Then:

(1) $\|h\|=\left\|h_{m}\right\|=\operatorname{Disc}\left(h_{m}\right)$. In particular, $\|h\| \in \mathbb{N}$.

(2) There exists a constant $C$ such that $n\|h\|-C \leq \operatorname{Disc}\left(h^{n}\right) \leq n\|h\|+C$ for all $n \geq 0$. 
(3) If $h^{\prime}$ is any other conjugate of $h$, then $\operatorname{Disc}\left(h_{m}^{n}\right) \leq \operatorname{Disc}\left(h^{\prime n}\right)$ for all $n$.

(4) $\|h\|=0$ if and only if $h$ is conjugate to a virtual multi-rotation.

(5) If $h$ is divisible (i.e., if $h$ has roots of arbitrary order), then $\|h\|=0$.

Proof. The first assertion is clear.

Let $g \in \operatorname{IET}\left(\mathscr{D}, \mathscr{D}_{m}\right)$ conjugating $h$ to $h_{m}$. Then $\operatorname{Disc}\left(h^{n}\right) \leq \operatorname{Disc}(g)+$ $\operatorname{Disc}\left(h_{m}^{n}\right)+\operatorname{Disc}\left(g^{-1}\right)=\operatorname{Disc}(g)+n\|h\|+\operatorname{Disc}\left(g^{-1}\right)$. Similarly, we have $n\|h\|=$ $\operatorname{Disc}\left(h_{m}^{n}\right) \leq \operatorname{Disc}(g)+\operatorname{Disc}\left(h^{n}\right)+\operatorname{Disc}\left(g^{-1}\right)$. Assertion 2 follows.

For assertion 3, assume that $\operatorname{Disc}\left(h^{\prime n_{0}}\right)<\operatorname{Disc}\left(h_{m}^{n_{0}}\right)=n_{0}\|h\|$. Then $\left\|h^{\prime}\right\|=$ $\inf \left\{\frac{1}{n} \operatorname{Disc}\left(h^{\prime n}\right)\right\}<\|h\|$, a contradiction.

If $\|h\|=0$, then $\operatorname{Disc}\left(h_{m}\right)=0$, so $h_{m}$ is continuous, i.e., a virtual multi-rotation. Hence $h$ is conjugate to a virtual multi-rotation. The converse is obvious.

Finally, assume that $\|h\|>0$. Since $\left\|g^{k}\right\|=k\|g\|$ and $\|\cdot\|$ takes only integer values, $h$ has no root of order larger that $\|h\|$.

The following lemma shows that the orbit of $\Delta\left(h_{m}\right)$ is canonical.

Lemma 2.6. Let $h_{m}$ be such that $\operatorname{Disc}\left(h_{m}\right)=\left\|h_{m}\right\|$. Then the centralizer of $h_{m}$ preserves $h_{m}^{\mathbb{Z}}\left(\Delta\left(h_{m}\right)\right)$. Equivalently, if $\left[g, h_{m}\right]=1$, then for any $x \in \Delta\left(h_{m}\right)$ there exists $k \in \mathbb{Z}$ such that $g(x) \in h_{m}^{k}\left(\Delta\left(h_{m}\right)\right)$.

Proof. Fix $x \in \Delta\left(h_{m}\right)$. Since $\left\|h_{m}\right\|=\operatorname{Disc}\left(h_{m}\right)$, we have $\operatorname{Disc}\left(h_{m}^{i}\right)=i \operatorname{Disc}\left(h_{m}\right)$ for all $i>0$, so $\Delta\left(h_{m}^{i}\right)$ is the disjoint union $\Delta\left(h_{m}\right) \sqcup \cdots \sqcup h_{m}^{-(i-1)} \cdot \Delta\left(h_{m}\right)$. In particular, $x, \ldots, h_{m}^{-(i-1)}(x) \in \Delta\left(h_{m}^{i}\right)$.

Since $g$ commutes with $h_{m}^{i}$ for all $i>0$,

$$
\Delta\left(h_{m}^{i}\right)=\Delta\left(g^{-1} h_{m}^{i} g\right) \subset \Delta(g) \cup g^{-1} \cdot \Delta\left(h_{m}^{i}\right) \cup g^{-1} h_{m}^{-i} \cdot \Delta\left(g^{-1}\right) .
$$

In particular, $\Delta\left(h_{m}^{i}\right) \backslash g^{-1} \cdot \Delta\left(h_{m}^{i}\right)$ contains at most $2 \operatorname{Disc}(g)$ elements. It follows that for $i>2 \operatorname{Disc}(g)$ there is some $i_{0} \in\{0, \ldots, i-1\}$ such that $h_{m}^{-i_{0}}(x) \in g^{-1} \cdot \Delta\left(h_{m}^{i}\right)$. Hence, $g h_{m}^{-i_{0}}(x)$ lies in some $h_{m}^{-j} \cdot \Delta\left(h_{m}\right)$ for some $j \in\{0, \ldots, i-1\}$, and the lemma follows since $g$ and $h_{m}$ commute.

These considerations allow to slightly simplify Novak's result on centralizers.

Corollary 2.7 ([9]). It $h \in$ IET acts with dense orbits on $\mathscr{D}$ and $\|h\| \neq 0$, then the centralizer of $h$ is virtually cyclic.

Proof. By Proposition 2.4, we may assume that $\|h\|=\operatorname{Disc}(h)$. Denote by $Z$ the centralizer of $h$. By the previous lemma, $Z$ permutes the $h$-orbits of the points of $\Delta(h)$. Let $Z_{0} \subset Z$ be the finite index subgroup that preserves the $h$-orbit of each point of $\Delta(h)$. We claim that $Z_{0}=\langle h\rangle$. Indeed, consider $g \in Z_{0}, x \in \Delta(h)$ and $k$ such that $g(x)=h^{k} x$. Since $g$ commutes with $h$, we have $g\left(h^{i}(x)\right)=h^{k}\left(h^{i} x\right)$ for all $i \in \mathbb{Z}$. Since the orbit of $x$ is dense, it follows that $g=h^{k}$. 
The proof of Proposition 2.4 is better visualized in terms of suspensions. It is a direct consequence of Lemmas 2.11 and 2.12, whose proofs occupy next subsection.

2.3. Suspensions and construction of minimal models. Consider $h \in \operatorname{IET}(\mathscr{D})$. We now define its suspension (in a slightly non-standard way). Let $I_{1}, \ldots, I_{k}$ be the continuity intervals of $h$, i.e., the maximal connected sets on which $h$ is continuous. Each $I_{i}$ is either a circle, or a semi-open interval, closed on the left. Let $\bar{D}$ be the metric completion of $\mathscr{D}$, and $\bar{I}_{i}$ the closure of $I_{i}$ in $\bar{D}$. Note that $\left.h\right|_{I_{i}}$ extends by continuity to a map $\bar{h}_{i}: \bar{I}_{i} \rightarrow \bar{D}$. We denote by $J_{i}=h\left(I_{i}\right)$, which are the maximal connected sets on which $h^{-1}$ is continuous and by $\bar{J}_{i}=\bar{h}_{i}\left(\bar{I}_{i}\right)$ its closure in $\bar{D}$.

We consider bands $B_{i}=\bar{I}_{i} \times[0,1]$, which we glue on $\bar{D}$ using two maps $\varphi_{\varepsilon}: \bar{I}_{i} \times$ $\{\varepsilon\} \rightarrow \bar{D}$ defined for $\varepsilon=0,1$ as follows: $\varphi_{0}:(x, 0) \in \bar{I}_{i} \times\{0\} \mapsto x \in \bar{I}_{i}$ is just the inclusion, and $\varphi_{1}(x, 1) \in \bar{I}_{i} \times\{1\} \mapsto \bar{h}_{i}(x) \in \bar{J}_{i}$ is the extension of $\left.h\right|_{I_{i}}$. Since $\bar{I}_{i}$ can be a circle, bands can be annuli.

We denote by $\Sigma(h)$ (or simply $\Sigma$ ) the cellular complex obtained in this way. We call it the suspension of $h$. If we foliate bands by $\{x\} \times[0,1], \Sigma$ inherits a natural foliation such that for all $x \in \mathscr{D}, x$ lies in the same leaf as $h(x)$.

Since $I_{1} \sqcup \cdots \sqcup I_{k}$ is a partition of $\mathscr{D}$, every $x \in \mathscr{D}$ lies in exactly one interval $\bar{I}_{i}$ except if $h$ is discontinuous at $x$, in which case $x$ lies in the intersection of exactly two closed intervals $\bar{I}_{i}$. Similarly, any $x \in \mathscr{D}$ lies in exactly one $\bar{J}_{i}$ except if $h^{-1}$ is discontinuous at $x$.

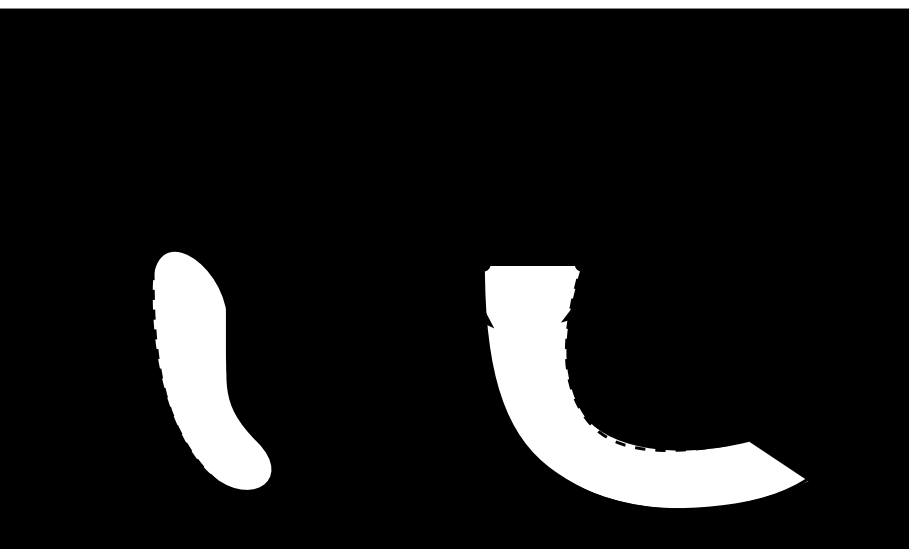

Figure 1. Suspension of an interval exchange transformation.

Denote by $\operatorname{Sing}(h)=\Delta(h) \cap \Delta\left(h^{-1}\right)$ the set of points where both $h$ and $h^{-1}$ are discontinuous (see Figure 1 where $\Delta(h)=\{b, d\}, \Delta\left(h^{-1}\right)=\{c, d\}$ and $\operatorname{Sing}(h)=$ $\{d\})$. In Figure 1, dotted lines correspond to leaf segments $x \times[0,1]$ whose endpoints are not the image of each other under $h$ (only under $\bar{h}_{i}$ ). 
Points in $\mathscr{D} \backslash\left(\Delta(h) \cup \Delta\left(h^{-1}\right)\right)$ (resp. $\left.\Delta(h) \cup \Delta\left(h^{-1}\right) \backslash \operatorname{Sing}(h)\right)$ have an neighbourhood in $\Sigma$ homeomorphic to $\mathbb{R}^{2}$ (resp. to a $\mathbb{R}_{+} \times \mathbb{R}$ ). In particular, $\Delta(h)$ is a surface with boundary if (and only if) $\operatorname{Sing}(h)=\emptyset$. In this case, $\Delta(h) \cup \Delta\left(h^{-1}\right) \subset \partial \Sigma$.

It will be useful to perform some moves that change $h$ to a conjugate, and that allow to simplify $\Sigma$.

Consider $h \in \mathrm{IET}(\mathscr{D})$, and $x \in \check{D}^{\text {. Let }} \mathscr{D}^{\prime}$ be the domain obtained from $\mathscr{D}$ by cutting at $x$. More precisely, if the component of $\mathscr{D}$ containing $x$ is $[\alpha, \beta)$ with $\alpha<x<\beta$, we replace $[\alpha, \beta)$ by the two intervals $[\alpha, x)$ and $[x, \beta)$. Similarly, if the component of $\mathscr{D}$ containing $x$ is a circle $\mathbb{R} / l \mathbb{Z}$, we replace it by an interval $[x, x+l)$. We denote by $i: \mathscr{D}^{\prime} \rightarrow \mathscr{D}$ the natural inclusion $\left(i \in \operatorname{IET}\left(\mathscr{D}^{\prime}, \mathscr{D}\right)\right)$, and define $h^{\prime}=i^{\prime-1} \circ h \circ i \in \operatorname{IET}\left(\mathscr{D}^{\prime}\right)$. We say that $h^{\prime}$ is obtained from $h$ by splitting $\mathscr{D}$ at $x$.

Lemma 2.8. Every $h \in \operatorname{IET}(D)$ is conjugate to some $h^{\prime} \in \operatorname{IET}\left(D^{\prime}\right)$ such that $\Sigma\left(h^{\prime}\right)$ is a surface with boundary.

Proof. Assume that $\Sigma(h)$ is not a surface and consider a point $x \in \operatorname{Sing}(h)$. Let $h^{\prime} \in$ $\operatorname{IET}\left(\mathscr{D}^{\prime}\right)$ be obtained by splitting $\mathscr{D}$ at $x$. We claim that \# $\operatorname{Sing}\left(h^{\prime}\right)=\# \operatorname{Sing}(h)-1$. The Lemma will follow by induction.

Denote by $i: \mathscr{D}^{\prime} \rightarrow \mathscr{D}$ the splitting map and by $x^{\prime}=i^{-1}(\{x\})$ the copy of $x$ in $\mathscr{D}^{\prime}$. Since $i$ is continuous on $\mathscr{D}^{\prime}$ and $i^{-1}$ is only discontinuous at $x, \operatorname{Sing}(h) \backslash x=$ $i\left(\operatorname{Sing}\left(h^{\prime}\right) \backslash\left\{x^{\prime}\right\}\right)$. Since $x^{\prime} \in \mathscr{D}^{\prime} \backslash \stackrel{\circ}{D}^{\prime}, h^{\prime}$ and $h^{\prime-1}$ are continuous at $x^{\prime}$. The claim follows.

When $\Sigma$ is a surface, one can describe its boundary $\partial \Sigma$. Each connected component of $\partial \Sigma$ is a circle $C$, which has a natural set of vertices $C \cap \mathscr{D}$, and a natural set of edges contained in boundaries of bands $\left(\bar{I}_{i} \backslash \stackrel{\circ}{I}_{i}\right) \times[0,1]$. Each edge $\{x\} \times[0,1]$ of $C$ carries a preferred orientation from 0 to 1 . A vertex $x \in C$ can be of three exclusive types: $x \in \bar{D} \backslash \grave{D}$ having one incoming and one outgoing edge (the vertices $a, e$ in Fig. 1), $x \in \Delta(h)$ having two outgoing edges (the vertex $b$ ), and $x \in \Delta\left(h^{-1}\right)$ having two incoming edges (the vertex $c$, recall that $\operatorname{Sing}(h)=\Delta(h) \cap \Delta\left(h^{-1}\right)=\emptyset$ by assumption).

We say that $\Sigma$ has a boundary connection if there exists a leaf segment in $\Sigma$ which intersects $\partial \Sigma$ exactly at its endpoints, or if $\operatorname{Sing}(h) \neq \emptyset$. Equivalently, $\Sigma(h)$ has a boundary connection if there exists $x \in \Delta\left(h^{-1}\right)$ and $k \geq 0$ such that $h^{k}(x) \in \Delta(h)$. Note that taking $k$ as small as possible, this is equivalent to ask that $h^{-1}$ discontinuous at $x$ but continuous at $h(x), h^{2}(x), \ldots, h^{k}(x)$ and $h$ discontinuous at $h^{k}(x)$ but continuous at $x, h(x), \ldots, h^{k-1}(x)$.

Lemma 2.9. Every $h \in \operatorname{IET}(D)$ is conjugate to some $h^{\prime} \in \operatorname{IET}\left(D^{\prime}\right)$ such that $\Sigma\left(h^{\prime}\right)$ has no boundary connection.

Proof. We can assume that $\operatorname{Sing}(h)=\emptyset$ by Lemma 2.8. Assume that $x, h(x)$, $h^{2}(x), \ldots, h^{k}(x)$ is a boundary connection, with $x \in \Delta\left(h^{-1}\right), h^{k}(x) \in \Delta(h)$, and 
$h(x), \ldots, h^{k-1}(x) \in D_{D} \backslash\left(\Delta(h) \cup \Delta\left(h^{-1}\right)\right)$. We split $\mathscr{D}$ at the points $x, h(x)$, $\ldots, h^{k}(x)$ and denote by $\mathscr{D}^{\prime}$ the new domain, and write $i: \mathscr{D}^{\prime} \rightarrow \mathscr{D}$ induced by inclusion. We write $x_{i}=h^{i}(x) \in \mathscr{D}$ and $x_{i}^{\prime}=i^{-1}\left(h^{i}(x)\right) \in \mathscr{D}^{\prime}$ for $i=0, \ldots, k$. Since $x_{i}^{\prime} \in \mathscr{D}^{\prime} \backslash \stackrel{\circ}{D}^{\prime}$, we have that $\Delta\left(h^{\prime}\right)$ contains no $x_{i}^{\prime}$. Moreover, since $i$ and $i^{-1}$ are continuous away from $x_{i}, x_{i}^{\prime}$, it follows that $i^{-1}\left(\Delta(h) \backslash\left\{x_{0}, \ldots, x_{k}\right\}\right)=$ $\Delta\left(h^{\prime}\right) \backslash\left\{x_{0}^{\prime}, \ldots, x_{k}^{\prime}\right\}=\Delta\left(h^{\prime}\right)$, and similarly for $\Delta(h)^{-1}$. We still have $\operatorname{Sing}\left(h^{\prime}\right)=\varnothing$ and $\Sigma\left(h^{\prime}\right)$ has one less boundary connection. By induction, the lemma follows.

Now there are some useless boundary components which we want to get rid of. They correspond to points $x$ such that $h$ is discontinuous at $x$, but some positive power is continuous at $x$. We will denote by $h\left(x^{-}\right)=\lim _{\varepsilon \rightarrow 0^{+}} h(x-\varepsilon)$ (only defined if $x \in \stackrel{D}{)})$ and by $h\left(x^{+}\right)=\lim _{\varepsilon \rightarrow 0^{+}} h(x+\varepsilon)=h(x)$.

Let $C \subset \partial \Sigma$ be a boundary component. We say that $C$ is a fake boundary if

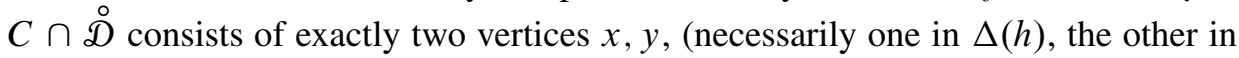
$\Delta\left(h^{-1}\right)$ ), and the two connected components of $C \backslash\{x, y\}$ have the same number of edges. Denote by $k$ this number of edges, and up to exchanging $x$ and $y$, assume $x \in C \cap \Delta(h)$. Then $h^{k}(x)$ coincides with $h^{k}\left(x^{-}\right)$, so $h^{k}$ is continuous at $x$ although $h$ is not.

Lemma 2.10. Assume that $\Sigma(h)$ has no boundary connection, and that $x \in \Delta(h)$ is such that $h^{k}$ is continuous $x$ for some $k>0$. Then $\Sigma(h)$ has a fake boundary.

Proof. Define $x_{i}^{+}=h^{i}(x)=h^{i}\left(x^{+}\right)$and $x_{i}^{-}=h^{i}\left(x^{-}\right)$, and let $k>0$ be smallest such that $x_{k}^{+}=x_{k}^{-}$. Since $x \in \Delta(h), k \geq 2$.

We claim that for all $i \leq k, x_{i}^{+}$and $x_{i}^{-}$lie in $\partial \Sigma(h)$. Otherwise, consider some $x_{i}^{+}$(resp. $x_{i}^{-}$) which does not lie in $\partial \Sigma(h)$. Then because there is no boundary connection, $x_{k}^{+}$(resp. $x_{k}^{-}$) does not lie in $\partial \Sigma(h)$, so $h^{-1}$ is continuous at $x_{k}^{+}=x_{k}^{-}$. It follows that $x_{k-1}^{+}=x_{k-1}^{-}$, a contradiction.

It follows that the component $C$ of $\partial \Sigma$ containing $x$ contains oriented edges joining $x_{i}^{+}$to $x_{i+1}^{+}\left(\right.$resp. $x_{i}^{-}$to $x_{i+1}^{-}$) for $i=0, \ldots, k-1$, and $C$ is a fake boundary.

Lemma 2.11. Every $h \in \operatorname{IET}(D)$ is conjugate to some $h_{m} \in \operatorname{IET}\left(\mathscr{D}_{m}\right)$ such that $\Sigma\left(h_{m}\right)$ has no boundary connection and no fake boundary.

Proof. First, by Lemma 2.9 we can assume that $\Sigma(h)$ has no boundary connection. We will produce a sequence of conjugates of $h$ whose suspensions have no boundary connection, and fewer fake boundaries. By finiteness of the number of boundaries, this will ensure the result.

Assume that $C$ is a fake boundary, denote by $C_{l}, C_{r}$ the two components of

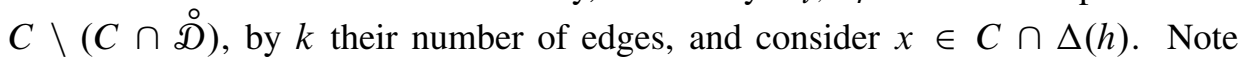
that $k \geq 2$ since otherwise $h$ would be continuous at $x$, a contradiction. Introduce 
$x_{i}=h^{i}(x) \in C_{r}$ for $i=\{1, \ldots, k-1\}$, and $y_{i}=h^{i}\left(x^{-}\right) \in C_{l}$. Note that $x_{i}$ is a (left) endpoint of $\mathcal{D}$, but $y_{i} \in \bar{D} \backslash \mathscr{D}$.

We now perform a gluing move on $h$ as follows. Let $\mathscr{D}^{\prime}$ be obtained from $\mathscr{D} \cup$ $\left\{y_{1}, \ldots, y_{k-1}\right\} \subset \bar{D}$ by identifying $y_{i}$ with $x_{i}$. Clearly, $\mathscr{D}^{\prime}$ is a domain, and the inclusion $j: \mathscr{D} \rightarrow \mathscr{D}^{\prime}$ allows to define $h^{\prime}=j h j^{-1} \in \operatorname{IET}\left(\mathscr{D}^{\prime}\right)$.

This is a general construction, and we claim that if $\Sigma(h)$ has no boundary connection, then neither does $\Sigma\left(h^{\prime}\right)$. Indeed assume that $x, h^{\prime}(x), \ldots,\left(h^{\prime}\right)^{k}(x)$ is a boundary connection in $\Sigma\left(h^{\prime}\right)$. Then if no $j^{-1}\left(\left(h^{\prime}\right)^{i}(x)\right) \in C(i=0, \ldots, k)$, its image under $j^{-1}$ would also be one in $\Sigma(h)$; and if some $j^{-1}\left(\left(h^{\prime}\right)^{i}(x)\right) \in C$, then a shorter path would provide a boundary connection in $\Sigma(h)$.

Since $\Sigma\left(h^{\prime}\right)$ has one less fake boundary, the lemma follows by induction.

Lemma 2.12. Let $h_{m}$ be as above. Then $\operatorname{Disc}\left(h_{m}^{n}\right)=n \operatorname{Disc}\left(h_{m}\right)$ for all $n \in \mathbb{N}$.

Proof. We can assume that $n>0$. By Lemma 2.2, one has $\operatorname{Disc}\left(h_{m}^{n}\right) \leq n \operatorname{Disc}\left(h_{m}\right)$.

Let $\Delta \subset \mathscr{D}$ be the set of discontinuity points of $h_{m}$. Recall that $\Delta$ lies in the boundary of the suspension $\Sigma$ of $h_{m}$. Let $x \in \Delta$. By Lemma 2.10, $h_{m}^{k}$ is discontinuous at $x$ for all $k>0$. We claim that $h_{m}^{n}$ is discontinuous at each point $h_{m}^{-k}(x)$ for $k=0, \ldots, n-1$. Indeed, since there is no boundary connection, $h_{m}^{-i}(x) \notin \partial \Sigma$ for all $i>0$, so $h_{m}^{-1}$ is continuous at all these points. If $h_{m}^{n}$ were continuous at $h_{m}^{-k}(x)$ $0 \leq k<n$, then $h_{m}^{n} \circ\left(h_{m}^{-1}\right)^{k}=h_{m}^{n-k}$ would be continuous at $x$, a contradiction.

We proved that $h_{m}^{n}$ is discontinuous on $\bigcup_{i=0}^{n} h_{m}^{-i}(\Delta)$. We claim that this is a disjoint union. Indeed, the absence of boundary connection implies that $h_{m}^{-i}(x)$ is not in $\partial \Sigma$, hence not in $\Delta$ for all $i>0$. It follows that all $h_{m}^{-i}(\Delta)$ are disjoint, and the lemma follows.

Proposition 2.4 is proved.

\section{Commutation relations involving multi-rotations}

Let $\mathcal{D}$ be a domain. For a subset $Y \subset \mathcal{D}$ and $\varepsilon>0$, let $Y_{\varepsilon}$ denote the closed $\varepsilon$ neighborhood of $Y$ in $\mathscr{D}$, where we assume that the distance between two components of $\mathscr{D}$ is infinite. We define $\operatorname{int}_{\varepsilon}(Y)=\{x \in Y \mid[x-\varepsilon, x+\varepsilon] \subset Y\}$. By definition, we have int $_{\varepsilon}(\mathcal{D} \backslash Y) \cap Y_{\varepsilon}=\emptyset$.

Recall that the support of $T \in \operatorname{IET}(\mathcal{D})$ is $\operatorname{supp}(T)=\{x \in \mathscr{D} \mid T(x) \neq x\}$. We denote by $[g, h]=g^{-1} h^{-1} g h$ the commutator of two group elements $g, h$. The following proposition allows to produce elements with small support.

Proposition 3.1. Let $R \in \operatorname{IET}(D)$ be a multi-rotation, and consider any $S \in$ $\operatorname{IET}(\mathscr{D})$. Let $X=(\bar{D} \backslash \grave{D}) \cup \Delta(S) \cup \Delta\left(S^{-1}\right)$. Then for all $\varepsilon>0$ there exists $n \geq 1$ such that the support of $U=\left[\left[S, R^{n}\right], R^{n}\right]$ is contained in $X_{\varepsilon}$, the closed $\varepsilon$-neighbourhood of $X$. 
Remark 3.2. If $\mathcal{D}$ is a union of circles, then $X=\Delta(S) \cup \Delta\left(S^{-1}\right)$.

We first prove a few easy lemmas. We will be concerned with subsets $E$ that consist of a finite union of sub-intervals.

Lemma 3.3. Consider a subset $E \subset \mathcal{D}, g \in \mathrm{IET}(\mathcal{D})$ such that $g$ is continuous (i.e., a translation) on each connected component of $E$. Consider $h \in \operatorname{IET}(\mathcal{D})$ whose restriction to each component of $E$ and of $g(E)$ is a (continuous) translation of amplitude $\in[-\varepsilon, \varepsilon]$.

Then on each component of $\operatorname{int}_{2 \varepsilon}(E)$, the restriction of $[g, h]$ is a translation of amplitude $\in[-2 \varepsilon, 2 \varepsilon]$.

Proof. Let $I$ be a connected component of $E$, and $t, t^{\prime} \in[-\varepsilon, \varepsilon]$ be such that $\left.h\right|_{I}(x)=$ $x+t$ and $\left.h\right|_{g(I)}(x)=x+t^{\prime}$. Then $h(x)=x+t \in \operatorname{int}_{\varepsilon}(I)$ for $x \in \operatorname{int}_{2 \varepsilon}(I)$, and since $\left.g\right|_{I}$ commutes with translations, $h^{-1} g h(x)=h^{-1}(g(x)+t)=g(x)+t-t^{\prime}$. Similarly, since $g(x)+t-t^{\prime} \in g(I)$, we have $g^{-1} h^{-1} g h(x)=x+t-t^{\prime}$.

As an immediate corollary we have:

Lemma 3.4. Let $g \in \mathrm{IET}(\mathcal{D})$ be arbitrary and $r \in \operatorname{IET}(\mathcal{D})$ a multi-rotation which moves all points of $\mathcal{D}$ by at most $\varepsilon / 2$. Let $X=\Delta(g) \cup \Delta\left(g^{-1}\right)$.

Then the restriction of $[g, r]$ to each component of $\operatorname{int}_{\varepsilon}(\mathscr{D} \backslash X)$ is a (continuous) translation of amplitude in $[-\varepsilon, \varepsilon]$.

Lemma 3.5. Let $E \subset \mathcal{D}$. Consider $g, h \in \mathrm{IET}(\mathcal{D})$ whose restrictions on each component of $E$ are (continuous) translations of amplitude $\in[-\varepsilon, \varepsilon]$.

Then $[g, h]$ is the identity in restriction to $\operatorname{int}_{\varepsilon}(E)$.

Proof. Let $x \in \operatorname{int}_{\varepsilon}(E)$. Denote by $\alpha, \beta$ the amplitude of the translations induced by $g$ and $h$ in the component of $E$ containing $x$. Since $g(x), h(x) \in E$, it follows that $h g(x)=x+\alpha+\beta=g h(x)$. The lemma follows.

Proof of Proposition 3.1. Let $R$ be a multi-rotation on a domain $D$ with $k$ circles. We view $R$ as an element of the torus group $\left(\mathbb{S}^{1}\right)^{k}$. Since this group is compact, powers of $R$ get arbitrarily close to the identity. In other words, for all $\varepsilon>0$ there exists $n$ such that $R^{n}$ moves all points of $\mathcal{D}$ by at most $\varepsilon / 2$. By Lemma 3.4, the restriction of $\left[S, R^{n}\right]$ to each component of $\operatorname{int}_{\varepsilon}(\mathscr{D} \backslash X)$ is a translation of amplitude in $[-\varepsilon, \varepsilon]$. By Lemma 3.5, $\left[\left[S, R^{n}\right], R^{n}\right]$ is the identity in restriction to $\operatorname{int}_{2 \varepsilon}(\mathscr{D} \backslash X)$. The proposition is proved.

Theorem 3.6. A non-abelian free subgroup of IET(D) contains no non-trivial element conjugate to a virtual multi-rotation. 
Proof. We argue by contradiction. Consider $R$ conjugate to a non-trivial virtual multirotation, contained in a non-abelian free subgroup $H$ of IET. Up to conjugating $H$ to a group of IETs on some other domain, we can assume that $R$ is indeed a virtual multi-rotation of $\mathscr{D}$. Since $R$ has infinite order, we can change $R$ to some power and assume that $R$ is an irrational multi-rotation. Consider $S \in H$ not commuting with $R$, so that $R, S$ form a basis of the subgroup they generate (if two elements generate a non-abelian free group, they are a basis of this free group by the Hopf property of free groups). Define $R^{\prime}=S R S^{-1}$ and note that $\left\{R, R^{\prime}\right\}$ freely generates a free subgroup of $H$. We aim to find a non-trivial relation between $R, R^{\prime}$ to get a contradiction.

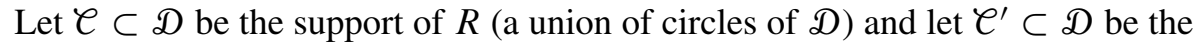
support of $R^{\prime}$ (which may be arbitrary). Up to restricting to a subdomain of $\mathscr{D}$, we can assume that $\mathcal{C} \cup \mathcal{C}^{\prime}=\mathscr{D}$, and we can still assume that $\mathscr{D}$ is a union of circles by identifying each interval of $\mathscr{D}$ (on which $R$ is the identity) to a circle of same length. Define $X=\Delta\left(R^{\prime}\right) \cup \Delta\left(R^{\prime-1}\right)$, and let $X_{\varepsilon}$ be its closed $\varepsilon$-neighbourhood.

Lemma 3.7. There exists $p, p^{\prime} \geq 1$ and $\varepsilon>0$ such that $R^{\prime p^{\prime}} R^{p}\left(X_{\varepsilon}\right) \cap X_{\varepsilon}=\emptyset$.

Proof. Since $\mathcal{C}$ is the support of the irrational multi-rotation $R$, there exists $p \geq 1$ such that $R^{p}(X \cap \mathcal{C}) \cap X=\emptyset$. Denote by $Y_{\varepsilon}=R^{p}\left(X_{\varepsilon}\right)=N_{\varepsilon}\left(R^{p}(X)\right)$ since $R$ is continuous. For $\varepsilon$ small enough, $Y_{\varepsilon} \cap \mathcal{C}$ does not intersect $X_{\varepsilon}$. We need to prove that $R^{\prime p^{\prime}} Y_{\varepsilon} \cap X_{\varepsilon}=\emptyset$ for suitable $p^{\prime}$ and $\varepsilon$. Since $R^{\prime}$ is the identity on $\mathscr{D} \backslash \mathcal{C}^{\prime}$ and since $\mathscr{D} \backslash \mathcal{C}^{\prime} \subset \mathcal{C}$, we have $R^{\prime p^{\prime}}\left(Y_{\varepsilon}\right) \cap\left(\mathscr{D} \backslash \mathcal{C}^{\prime}\right) \subset Y_{\varepsilon} \cap \mathcal{C}$ for any $p^{\prime}$, which does not intersect $X_{\varepsilon}$. Thus, we need only to prove that $R^{\prime p^{\prime}}\left(Y_{\varepsilon}\right) \cap \mathcal{C}^{\prime}$ does not intersect $X_{\varepsilon}$ for suitable $p^{\prime}$ and $\varepsilon$, or equivalently (since $\left.R^{\prime}=S R S^{-1}\right)$, that $R^{p^{\prime}}\left(S^{-1}\left(Y_{\varepsilon}\right) \cap \mathcal{C}\right.$ ) does not intersect $S^{-1}\left(X_{\varepsilon}\right)$. Now there exist finite sets $X^{\prime}, Y^{\prime} \subset \mathbb{D}$ such that, for all $\varepsilon>0$ small enough, $S^{-1}\left(X_{\varepsilon}\right)$ and $S^{-1}\left(Y_{\varepsilon}\right)$ are contained in the $\varepsilon$-neighbourhood of $X^{\prime}$ and $Y^{\prime}$ respectively. Denote by $X_{\varepsilon}^{\prime}, Y_{\varepsilon}^{\prime}$ these neighbourhoods. As above, since $X^{\prime}$ and $Y^{\prime}$ are finite, there exists $p^{\prime}$ and $\varepsilon$ such that $R^{p^{\prime}}\left(Y^{\prime} \cap \mathcal{C}\right)$ does not intersect $X^{\prime}$, so $R^{p^{\prime}}\left(Y_{\varepsilon}^{\prime} \cap \mathcal{C}\right)$ does not intersect $X_{\varepsilon}^{\prime}$ for $\varepsilon$ small enough. This proves the lemma.

We are ready to conclude the proof of Theorem 3.6 by exhibiting a non-trivial relation between $R$ and $R^{\prime}$. In view of Lemma 3.7, consider $p, p^{\prime} \geq 1$ and $\varepsilon>0$ such that $R^{\prime p^{\prime}} R^{p}\left(X_{\varepsilon}\right) \cap X_{\varepsilon}=\emptyset$. By Proposition 3.1, there exists $n \geq 1$ such that the support of $U=\left[\left[R^{\prime}, R^{n}\right], R^{n}\right]$ is contained in $X_{\varepsilon}$ (see Remark 3.2). Since $U$ and its conjugate under $R^{\prime p^{\prime}} R^{p}$ have disjoint supports, they commute. In other words, we have the relation $\left[U, R^{\prime p^{\prime}} R^{p} U R^{-p} R^{\prime-p^{\prime}}\right]=$ id.

One easily checks that this relator is non-trivial, i.e., that $\left[U, R^{\prime p^{\prime}} R^{p} U R^{-p} R^{\prime-p^{\prime}}\right]$, viewed as an element of the free group $F$ freely generated by $R, R^{\prime}$, is non-trivial in $F$. First $\left[R^{\prime}, R^{n}\right]$ is non-trivial in $F$. Since commuting elements of $F$ are powers of a common element [7], Prop. I.2.17-18, we see that $R^{n}$ does not commute with $\left[R^{\prime}, R^{n}\right]$, so $U \neq 1$. The normalizer of the maximal cyclic subgroup containing $U$ in $F$ being cyclic [7], Prop. I.2.19, we get that $\left[U, R^{\prime p^{\prime}} R^{p} U R^{-p} R^{-p^{\prime}}\right]=1$ would 
imply $\left[U, R^{\prime p^{\prime}} R^{p}\right]=1$ in $F$, which is impossible since $U$ and $R^{\prime p^{\prime}} R^{p}$ are not powers of a common element. Theorem 3.6 is proved.

\section{Application to Lie groups}

Consider a connected Lie group $L$. Recall that the exponential map of a Lie group is a local diffeomorphism from the Lie algebra of the group, in the group. Any element in its image belongs to a one-parameter subgroup, and therefore admits roots of arbitrary order (in other words, it is infinitely divisible). See for instance [12], Chap. 2, Proposition 3.2.

Recall also that there exists a unique maximal connected solvable normal Lie subgroup, $\operatorname{Rad} L$, called the radical of $L$, and $L / \operatorname{Rad} L$ is semisimple (see [12], Chap. 2, Theorem 5.11, for instance). On the other hand, every non-trivial semisimple Lie group contains a non-abelian free subgroup that is dense [6], final Corollary. In particular there are free subgroups of $L$ generated by elements arbitrarily close to the identity. ${ }^{1}$

Proposition 4.1. Let $L$ be a connected Lie group. If $L \hookrightarrow$ IET is an injective homomorphism (not necessarily continuous), then $L$ is solvable.

Proof. Assume that $L$ is not solvable and that $L$ embeds in IET. Then $L$ contains a non-abelian free group generated by elements $a, b$ in the image of the exponential map, hence divisible. By Corollary 2.5 (4), (5), the image of $a$ and $b$ in IET are conjugate to virtual multi-rotations. This contradicts Theorem 3.6.

Proposition 4.2. Let $\mathcal{A}$ be an abelian connected Lie group and $\mathcal{B} \simeq \mathbb{R}$ or $\mathcal{B} \simeq \mathbb{S}^{1}$. Let $L$ be a Lie group that is an extension (of Lie groups) $1 \rightarrow \mathcal{A} \rightarrow L \rightarrow \mathscr{B} \rightarrow 1$ in which $\mathcal{A}$ is closed.

Assume that $L \hookrightarrow$ IET. Then $L$ is abelian.

Remark 4.3. We do not assume that the embedding in IET is continuous. However, the maps occurring in the extension are continuous, and $L \rightarrow \mathscr{B}$ is a surjective submersion.

Proof. We identify the elements of $L$ with their images in IET. Density arguments below take place in the Lie group.

Write $\mathcal{A} \simeq \mathbb{R}^{n} \times\left(\mathbb{S}^{1}\right)^{m}$, and choose a finite subset $F \subset \mathcal{A}$ generating a dense subgroup. Note that by Corollary 2.5 (5), every element of $\mathcal{A}$ is conjugate to a virtual multi-rotation. Replacing every element of $F$ by a power, $\langle F\rangle$ remains dense in $\mathcal{A}$. Thus, we can assume that elements of $F$ are conjugate to irrational multi-rotations.

\footnotetext{
${ }^{1}$ Note that this argument using [6] can be replaced by the genericity of free subgroups in $L$, provided there is one free subgroup to begin with, as recalled in the introduction
} 
First we claim that there exist a pair of commuting elements $Q_{1}, Q_{2} \in L$ both conjugate to irrational multi-rotations, whose image generates a dense subgroup in $\mathscr{B}$ (if $\mathcal{B} \simeq \mathbb{S}^{1}$, we only need one element, but if $\mathcal{B} \simeq \mathbb{R}$ we clearly need a pair). Indeed, consider two elements $b_{1}, b_{2} \in \mathscr{B}$ of infinite order, generating a dense subgroup of $\mathcal{B}$. One can choose $b_{1}, b_{2}$ close enough to the identity so that they have preimages $Q_{1}, Q_{2} \in L$ in the image of the exponential map. One can choose $Q_{1}, Q_{2}$ in the same one-parameter subgroup, so $Q_{1}$ commutes with $Q_{2}$. By Corollary 2.5 (4), (5), $Q_{1}, Q_{2}$ are conjugate to virtual multi-rotations. Replacing them by powers, we can assume that $Q_{1}, Q_{2}$ are conjugate to irrational multi-rotations.

Since $\mathcal{A}$ is normal and abelian, for each $T \in F$ and each $Q_{i}, Q_{i}^{n} T Q_{i}^{-n}$ commutes with $T$ for all $n>0$. By Lemma 1.5, $T$ commutes with $Q_{i}$. Since $\langle F\rangle$ is dense in $\mathcal{A}$, $Q_{i}$ commutes with $\mathcal{A}$. Since $Q_{1}$ commutes with $Q_{2},\left\langle Q_{1}, Q_{2}, \mathcal{A}\right\rangle$ is abelian. Since $\left\langle Q_{1}, Q_{2}, \mathcal{A}\right\rangle \mathcal{A}$ is dense in $L, L$ is abelian.

Theorem 4.4. If $L$ is a connected Lie group and $L \hookrightarrow$ IET is an injective homomorphism, then $L$ is abelian.

Proof. By Proposition 4.1, $L$ is solvable. Assume it is not abelian, and write $\{1\}=$ $L_{n+1} \triangleleft L_{n} \triangleleft L_{n-1} \triangleleft \cdots \triangleleft L_{1}=L$ where $L_{i+1}=\left[L_{i}, L_{i}\right]$. One can assume $n$ to be minimal, that is, $L_{n-1}$ non-abelian.

By induction, we see that each $L_{i}$ is connected. In particular, the closures $\overline{L_{n}}$ and $\overline{L_{n-1}}$ are connected Lie subgroups of $L$. Since $L_{n}$ is abelian, so is $\overline{L_{n}}$. Moreover, since $\left[L_{n-1}, L_{n-1}\right] \subset L_{n},\left[\overline{L_{n-1}}, \overline{L_{n-1}}\right] \subset \overline{L_{n}}$.

This means that $\overline{L_{n-1}} / \overline{L_{n}}$ is a connected abelian Lie group, hence isomorphic to $\mathbb{R}^{k} \times\left(\mathbb{S}^{1}\right)^{j}$. Hence $\overline{L_{n-1}}$ is an extension (of Lie groups) $1 \rightarrow \overline{L_{n}} \rightarrow \overline{L_{n-1}} \stackrel{\pi}{\rightarrow}$ $\mathbb{R}^{k} \times\left(\mathbb{S}^{1}\right)^{j} \rightarrow 1$.

Let us write $\mathbb{R}^{k} \times\left(\mathbb{S}^{1}\right)^{j} \simeq \bigoplus_{i \leq k+j} \mathcal{B}_{i}$, coordinate by coordinate. Since $\overline{L_{n-1}}$ itself is non-abelian, there exists a least index $i_{0}$ such that $\pi^{-1}\left(\bigoplus_{i \leq i_{0}} \mathscr{B}_{i}\right)$ is not abelian. Note that $\pi^{-1}(\{1\})=\overline{L_{n}}$ is abelian, so that $i_{0} \geq 1$.

Consider $\pi^{-1}\left(\bigoplus_{i \leq i_{0}-1} \mathcal{B}_{i}\right)$ (if $i_{0}=1$ this is $\left.\overline{L_{n}}\right)$. It is abelian, by definition of $i_{0}$, normal and closed in $\pi^{-1}\left(\bigoplus_{i \leq i_{0}} \mathcal{B}_{i}\right)$, as it is the kernel of the natural projection $\pi^{-1}\left(\bigoplus_{i \leq i_{0}} \mathscr{B}_{i}\right) \rightarrow \mathcal{B}_{i_{0}}$. By Proposition 4.2, $\pi^{-1}\left(\bigoplus_{i \leq i_{0}} \mathscr{B}_{i}\right)$ does not embed in IET, and neither does $L$.

\section{Generic subgroups are not free}

The main result of this section says that under some irreducibility assumption, a "generic" subgroup of IET is not free. This genericity is defined in topological terms using the natural topology on IET where varying the lengths of the intervals of continuity of an IET with fixed combinatorics describes a simplex (see below or [5], Chap. 14.5, for a formal definition). Note however that group operations are not continuous in this topology. 
More precisely, if $I_{1}, \ldots, I_{n}$ (resp. $J_{1}, \ldots, J_{n}$ ) are the continuity intervals of $T$ (resp. $T^{-1}$ ), ordered in an increasing fashion, then the underlying permutation $\sigma$ is the permutation of $\{1, \ldots, n\}$ so that $T\left(I_{i}\right)=J_{\sigma(i)}$. We denote by $\mathrm{IET}_{\sigma}$ the set of all interval exchange transformations (with $n-1$ points of discontinuity) whose underlying permutation is $\sigma$. Assigning to a transformation $T \in \mathrm{IET}_{\sigma}$ the $n$-tuple of lengths of its continuity intervals, yields a natural bijection of IET $\sigma$ with the open simplex $\Delta_{n-1}$ of dimension $n-1$. Clearly, $\sigma$ occurs as the permutation underlying some IET if and only if for all $i<n, \sigma(i+1) \neq \sigma(i)+1$, and we denote by $\mathbb{S}_{n}$ the set of such permutations. Thus IET $=\sqcup_{n \geq 1} \sqcup_{\sigma \in \mathbb{S}_{n}}$ IET $_{\sigma}$ and the topology we consider is the one for which each subset $\mathrm{IET}_{\sigma}$ is open and the bijection above with $\Delta_{n-1}$ is a homeomorphism.

Definition 5.1. The permutation $\sigma$ of $\{1, \ldots, n\}$ is admissible if there is no $m \in$ $\{1, \ldots, n\}$ such that $\sigma(m)=m$ and $\sigma$ preserves $\{1, \ldots, m-1\}$ (and therefore $\{m+$ $1, \ldots, n\})$. We denote by $\operatorname{IET}_{a} \subset \mathrm{IET}$ the set of all transformations whose underlying permutation is admissible.

The main result of this section is Theorem 5.2:

Theorem 5.2. There is a dense open set $\Omega_{a} \subset \mathrm{IET} \times \mathrm{IET}_{a}$ such that $\langle S, T\rangle$ is not free for all $(S, T) \in \Omega_{a}$.

Remark 5.3. In the statement, $S$ is not required to be in $\mathrm{IET}_{a}$.

5.1. Explanation in a simpler situation. Before going into more technical arguments, we sketch a proof of the analogous result in a simpler situation. Here, we consider interval exchange transformations on the circle $\mathbb{R} / \mathbb{Z}$. Denote by $R_{\theta}$ the rotation $x \mapsto x+\theta$ on $\mathbb{R} / Z$. There is a natural topology on $\operatorname{IET}(\mathbb{R} / \mathbb{Z})$ such that $R_{\theta} \circ T$ varies continuously with $\theta$. This possibility to drift $T$ will be important below. For instance, given a permutation $\sigma$ of $\{1, \ldots, n\}$ there is a natural set of IETs, parametrized by $\mathbb{R} / \mathbb{Z} \times \mathbb{R} / \mathbb{Z} \times \Delta_{n-1}$. The two factors $\mathbb{R} / \mathbb{Z}$ indicate the initial points of $I_{1}$ and $J_{1}$, and the simplex $\Delta_{n-1}$ defines the lengths of the intervals (note that in this setting the permutation $\sigma$ is not uniquely defined in terms of $T$, but only its double coset modulo the cycle $(1, \ldots, n))$.

Consider $S_{0}, T_{0}$ two IETs on $\mathbb{R} / \mathbb{Z}$ whose points of discontinuity are in the finite set $D_{q}=\left(\frac{1}{q} \mathbb{Z}\right) / \mathbb{Z} \subset \mathbb{R} / \mathbb{Z}$, and whose translation lengths are in $D_{q}$. Then $D_{q}$ is invariant under $S_{0}$, and $S_{0}^{q !}$ is the identity. If $S$ is a small perturbation of $S_{0}$, then $S^{q !}$ agrees with a very small translation on each interval that is contained in the complement of a small neighbourhood of $D_{q}$. Now consider $T$ a small perturbation of $T_{0}$. Since two translations commute, this implies that the support of the commutator $\left[S^{q !}, T S^{q !} T^{-1}\right]$ is contained in a small neighbourhood of $D_{q}$, say $N_{\varepsilon}\left(D_{q}\right)$ (Lemma 3.5).

Given $\theta>0$, define $T_{\theta}=R_{\theta} \circ T_{0}$, and consider $T$ a small perturbation of $T_{\theta}$. If $\theta$ is small enough, and if $T$ is close enough to $T_{\theta}$, the discussion above applies in 
particular to $T$. Note that all translation lengths of $T_{\theta}$ are equal to $\theta$ modulo $\frac{1}{q} \mathbb{Z}$. We view $\theta$ as a drift since modulo $\frac{1}{q} \mathbb{Z}, R_{\theta} \circ T_{0}$ moves points uniformly by $\theta$ to the right. Because of this drift, some power of $R_{\theta} \circ T_{0}$ sends $N_{\varepsilon}\left(D_{q}\right)$ disjoint from itself. If we perturb $T_{0}$ into a transformation $T$, all translation lengths of $T$ are very close to $\theta$ modulo $\frac{1}{q} \mathbb{Z}$ so $T$ still features this drift. Thus, if $T$ is close enough to $R_{\theta} \circ T_{0}$, some power of $T$ will also send $N_{\varepsilon}\left(D_{q}\right)$ disjoint from itself. This means that for some $k \neq 0$, the support of $\left[S^{q !}, T S^{q !} T^{-1}\right]$ will be disjoint from its image under $T^{k}$, so $\left[S^{q !}, T S^{q !} T^{-1}\right]$ commutes with its conjugate by $T^{k}$. This prevents $\langle S, T\rangle$ from being free.

In what follows, we run the same strategy, except that the drift is not given by composition by a rotation but by a suitably designed modification of the IET $T_{0}$.

5.2. Linear maps. Now we come back to $\operatorname{IET}([0,1))$. Given $\sigma \in \mathbb{S}_{n}$, one has a homeomorphism $\lambda$ : IET $_{\sigma} \rightarrow \Delta_{n-1}$ assigning to an IET $T$ the tuple $\lambda(T)=$ $\left(l_{1}, \ldots, l_{n}\right)$ of lengths of its intervals of continuity. This allows to define the $\ell^{1}$ metric on $\operatorname{IET}_{\sigma}$ by $d\left(T, T^{\prime}\right)=\left\|\lambda(T)-\lambda\left(T^{\prime}\right)\right\|_{1}=\sum_{i=1}^{n}\left|l_{i}-l_{i}^{\prime}\right|$. We extend this definition to IET by saying that two transformations with distinct underlying permutations are at infinite distance.

The tuple $\beta(T)=\left(b_{1}<\cdots<b_{n-1}\right)$ of points of discontinuity of $T$ is related to $\left(l_{1}, \ldots, l_{n}\right)$ by the formulae $l_{i}=b_{i}-b_{i-1}$ (with the obvious conventions $b_{0}=$ $\left.0, b_{n}=1\right)$ and $b_{i}=\sum_{j \leq i} l_{i}$. This implies that $\left|b_{i}-b_{i}^{\prime}\right| \leq d\left(T, T^{\prime}\right)$ for all $i$.

We will also be interested in the tuple of translation lengths of an IET: given a transformation $T \in \mathrm{IET}_{\sigma}$, define $\varphi(T)=\left(t_{1}, \ldots, t_{n}\right)$ where $t_{i}$ is the translation length of $T$ in restriction to its $i$-th continuity interval. The following is immediate.

Lemma 5.4. The map $\varphi \circ \lambda^{-1}:\left(l_{1}, \ldots, l_{n}\right) \mapsto\left(t_{1}, \ldots, t_{n}\right)$ is linear. Explicitly,

$$
t_{i}=-\sum_{j=1}^{i-1} l_{j}+\sum_{j=1}^{\sigma(i)-1} l_{\sigma^{-1}(j)}
$$

In particular, $\left|t_{i}-t_{i}^{\prime}\right| \leq 2 d\left(T, T^{\prime}\right)$ for all $i \in\{1, \ldots, n\}$.

5.3. $q$-rationality and small support. A transformation $S_{0} \in \operatorname{IET}([0,1))$ is called $q$-rational, for $q \in \mathbb{N}$, if all its discontinuity points are in $\frac{1}{q} \mathbb{N}$. Clearly, in such case, all its translation lengths are in $\frac{1}{q} \mathbb{Z}$ and $S_{0}^{q !}=$ id. We also note that a $q$-rational transformation has at most $q$ continuity intervals.

Lemma 5.5. For all $\varepsilon>0, q \in \mathbb{N}, m \in \mathbb{N}$, there exists $\eta>0$ such that if $S_{0}$ and $T_{0}$ are $q$-rational, if $w(s, t)$ is a word of length $\leq m$ in the letters $s^{ \pm 1}, t^{ \pm 1}$ such that $w\left(S_{0}, T_{0}\right)=\mathrm{id}$, and if $S$ and $T$ are $\eta$-close to $S_{0}$ and $T_{0}$ respectively, then on each interval of $[0,1) \backslash \mathcal{N}_{\varepsilon}\left(\frac{1}{q} \mathbb{N}\right)$ the transformation $w(S, T)$ induces a translation of length $<\varepsilon$. 
Proof. Let $\varepsilon>0$, and choose $\eta<\frac{\varepsilon}{2 m}$. Let $I=(a, b)$ be any connected component of $[0,1) \backslash \frac{1}{q} \mathbb{N}$. Points of discontinuity of $S$ (resp. $T$ ) are within $\eta$ from those of $S_{0}$ (resp. $T_{0}$ ). Moreover, in restriction to $(a+2 \eta, b-2 \eta), S$ and $S_{0}$ (resp. $T$ and $\left.T_{0}\right)$ are translations of amplitude differing by at most $2 \eta$ by Lemma 5.4 .

It follows that if $k \geq 1$, the endpoints of $S((a+2 k \eta, b-2 k \eta))$ avoid the $2(k-1) \eta$-neighborhood of $\frac{1}{q} \mathbb{N}$ (and similarly for $T$ ). This is enough to ensure, by induction, that on each component of $[0,1) \backslash \mathcal{N}_{2 m \eta}\left(\frac{1}{q} \mathbb{N}\right)$ the transformation $w(S, T)$ is a translation, and its amplitude differs from that of $w\left(S_{0}, T_{0}\right)$ by at most $2 m \eta$. Since $w\left(S_{0}, T_{0}\right)$ is the identity, the result follows.

Lemma 5.6. For all $\varepsilon>0, q \in \mathbb{N}$, there exists $\eta>0$ such that if $S_{0}$ and $T_{0}$ are $q$-rational, and if $S$ and $T$ are $\eta$-close to $S_{0}$ and $T_{0}$ respectively, then on each interval of $[0,1) \backslash \mathcal{N}_{\varepsilon}\left(\frac{1}{q} \mathbb{N}\right)$ the transformation $\left[S^{q !}, T S^{q !} T^{-1}\right]$ induces the identity.

Proof. Apply the previous lemma to the words $s^{q !}$ and $t s^{q !} t^{-1}$ for $\varepsilon / 4$. Then apply Lemma 3.5.

\subsection{Drifting the support}

5.4.1. Drift vector. In this section, we introduce the notion of drift vector, a substitute to the composition by a rotation that can be used over a circle.

Fix a permutation $\sigma$, and consider the linear map $\Phi_{\sigma}=\varphi \circ \lambda^{-1}$ that assigns to tuple of lengths $\left(l_{i}\right)_{i=1, \ldots, n}$ the tuple of translation lengths $\left(t_{i}\right)_{i=1, \ldots, n}$ (see Lemma 5.4).

Definition 5.7. We say that a permutation $\sigma \in \mathbb{S}_{n}$ is driftable if there exists a vector $\overrightarrow{d l}=\left(d l_{1}, \ldots, d l_{n}\right) \in \mathbb{R}^{n}$ with $\sum d l_{i}=0$ such that the vector $\overrightarrow{d r}=\Phi_{\sigma}(\overrightarrow{d l}) \in \mathbb{R}^{n}$ has only positive (non-zero) coordinates.

When it exists, we call $\overrightarrow{d r}$ a drift vector, and $\overrightarrow{d l}$ a drifting direction.

The vector $\overrightarrow{d r}=\Phi_{\sigma}(\overrightarrow{d l})$ represents the change of translation lengths induced by the change of lengths of intervals $\overrightarrow{d l}$. The point of a drift vector is therefore that all translation lengths increase when we change the lengths of the intervals by a positive multiple of $\overrightarrow{d l}$. In Proposition 5.12, we will see that $\sigma$ is driftable if and only if it is admissible.

In the remainder of this section we assume that the permutation $\tau$ is driftable, and we fix $\overrightarrow{d l}$ and $\overrightarrow{d r}$ as above.

Consider $T_{0} \in \mathrm{IET}_{\tau}$ a given $q$-rational transformation. Given $\theta>0$, define $T_{\theta} \in I E T_{\tau}$ in terms of lengths of its continuity intervals by $\lambda\left(T_{\theta}\right)=\lambda\left(T_{0}\right)+\theta \overrightarrow{d l}$.

Definition 5.8. We denote by $d r_{\max }=\max _{i=1}^{n} d r_{i}$ and $d r_{\min }=\min _{i=1}^{n} d r_{i}$ the maximal and minimal drift of $\overrightarrow{d r}$. 
Lemma 5.9. All translation lengths of $T_{\theta}$ are in $\left[\theta d r_{\min }, \theta d r_{\max }\right] \bmod \frac{1}{q}$.

Moreover, if $\left\|\lambda(T)-\lambda\left(T_{\theta}\right)\right\|_{1} \leq \mu$, then all translation lengths of $T$ are in $\left[\theta d r_{\min }-2 \mu, \theta d r_{\max }+2 \mu\right] \bmod \frac{1}{q}$.

Proof. All translation lengths of $T_{0}$ are $0 \bmod \frac{1}{q}$. By linearity of $\Phi_{\tau}$ (as defined at the beginning of Section 5.4.1, the translation vector of $T_{\theta}$ is $\Phi_{\tau}\left(\lambda\left(T_{0}\right)+\theta \overrightarrow{d l}\right)=$ $\Phi_{\tau}\left(\lambda\left(T_{0}\right)\right)+\theta \Phi_{\tau}(\overrightarrow{d l})=\varphi\left(T_{0}\right)+\theta \overrightarrow{d r}$. Therefore, the translation lengths of $T_{\theta}$ are the coordinates of $\theta \overrightarrow{d r}$ mod $\frac{1}{q}$. The first assertion follows.

For the second statement, express $\lambda(T)$ as $\lambda(T)=\lambda\left(T_{0}\right)+\theta \overrightarrow{d l}+\vec{\varepsilon}$, where $\|\vec{\varepsilon}\|_{1} \leq \mu$. By linearity, $\varphi(T)=\varphi\left(T_{0}\right)+\theta \overrightarrow{d r}+\Phi_{\tau}(\vec{\varepsilon})$, which mod $\frac{1}{q}$, gives $\theta \overrightarrow{d r}+\Phi_{\tau}(\vec{\varepsilon})$. Now, writing $\vec{\varepsilon}=\left(\varepsilon_{i}\right)_{i=1}^{n}$, we have by Lemma 5.4,

$$
\Phi_{\tau}(\varepsilon)=\left(-\sum_{j=1}^{i-1} \varepsilon_{j}+\sum_{j=1}^{\tau(i)-1} \varepsilon_{\tau^{-1}(j)}\right)_{i=1, \ldots, n} .
$$

Since $\|\vec{\varepsilon}\|_{1} \leq \mu$, every coordinate of this vector is at most $2 \mu$.

\subsubsection{Generic absence of free groups}

Proposition 5.10. Assume that $S_{0}, T_{0}$ are $q$-rational and that the permutation $\tau$ underlying $T_{0}$ is driftable.

Then there exist a neighbourhood $U$ of $S_{0}$ which accumulates on $T_{0}$ such that $\langle S, T\rangle$ is not free of rank 2 whenever $(S, T) \in \mathcal{U} \times \mathcal{V}$.

Remark 5.11. We do not claim that $\mathcal{V}$ is a neighbourhood of $T_{0}$, only that $T_{0} \in \overline{\mathcal{V}}$.

Proof. Let $\overrightarrow{d l}, \overrightarrow{d r}$ be a drifting direction and a drifting vector as above, and $\rho=\frac{d r_{\max }}{d r_{\min }}$. Let $\varepsilon<\min \left(\frac{1}{100 \rho}, \frac{1}{10 q}\right)$, and let $\eta$ be given by Lemma 5.6. Let $U$ be the set of transformations $S$ at distance $\eta$ from $S_{0}$.

Now consider $\theta>0$ such that $\theta d r_{\max } \leq \eta / 10, \theta\|\overrightarrow{d l}\|_{1} \leq \eta / 10$, and $\theta d r_{\min }<\varepsilon$. The transformation $T_{\theta}$ defined above is then at distance $\leq \eta / 10$ from $T_{0}$. Finally, let $\mu<\frac{\theta d r_{\min }}{4}, \mu<\eta / 2$. Let $\mathcal{V}_{\theta}$ be the set of tranformations $T \in \mathrm{IET}_{\tau}$ at distance $<\mu$ from $T_{\theta}$. Note that any $T \in \mathcal{V}_{\theta}$ is at distance $<\eta$ from $T_{0}$. In particular, any $(S, T) \in \mathcal{U} \times \mathcal{V}_{\theta}$ satisfies the assumptions of Lemma 5.6. It follows that $U=\left[S^{q !}, T S^{q !} T^{-1}\right]$ has support in the $\varepsilon$-neighborhood of $\frac{1}{q} \mathbb{N}$.

We claim that there exists $k$ such that $T^{k} U T^{-k}$ has support disjoint from that of $U$. The claim implies that $\left[T^{k} U T^{-k}, U\right]=\mathrm{id}$. As in the end of the proof of Theorem 3.6, one checks that this word is non-trivial (as an element of the abstract free group on $S, T)$, so $\langle S, T\rangle$ is not free group of rank 2 . Taking for $\mathcal{V}$ the union of all $\mathcal{V}_{\theta}$ for $\theta$ small enough concludes the proposition. 
Let us now prove the claim. It suffices to show that there exists $k$ such that all translation lengths of $T^{k}$ lies in $\left[2 \varepsilon, \frac{1}{q}-2 \varepsilon\right]$ modulo $\frac{1}{q} \mathbb{Z}$.

We know from Lemma 5.9 that all translation lengths of $T$ are in $\left[\theta d r_{\min }\right.$ $\left.2 \mu, \theta d r_{\max }+2 \mu\right] \bmod \frac{1}{q}$. Thus by our choice of $\mu$ they are in $\left[\theta d r_{\min } / 2,2 \theta d r_{\max }\right]$ $\bmod \frac{1}{q}$. Hence any translation length $\tau$ of $T^{k}$ satisfies $\tau \in\left[k \theta d r_{\min } / 2,2 k \theta d r_{\max }\right]+$ $\frac{1}{q} \mathbb{Z}$.

Since $\theta d r_{\min }<\varepsilon$, there exists $k$ such that $2 \varepsilon<k \theta d r_{\min } / 2 \leq 3 \varepsilon$. For such a $k$, one has $2 k \theta d r_{\max }=2 k \theta d r_{\min } \times \rho \leq 12 \varepsilon \rho$. Since $\varepsilon<\min \left(\frac{1}{100 \rho q}, \frac{1}{10 q}\right)$, one successively gets $12 \varepsilon \rho \leq \frac{12}{100 q}<\frac{1}{q}-2 \varepsilon$. This establishes the claim.

5.4.3. Driftable permutation. To conclude this section, we prove that a permutation is driftable if and only if it is admissible. Recall that $\sigma \in \widetilde{S}_{n}$ is driftable if there exists a change of lengths $\overrightarrow{d l}=\left(d l_{1}, \ldots, d l_{n}\right)$ with $\sum d l_{i}=0$ that increases all translation lengths of the corresponding IET (Definition 5.7), and that $\sigma$ is non-admissible if there is an $m \in\{1, \ldots, n\}$ such that $\sigma(m)=m$ and $\sigma(\{1, \ldots, m-1\})=\{1, \ldots, m-1\}$ (Definition 5.1).

Proposition 5.12. Let $\sigma \in \mathbb{S}_{n}$. Then $\sigma$ is driftable if and only if it is admissible.

Let us note that, together with Proposition 5.10, this immediately implies Theorem 5.2.

Before proving proposition 5.12, we treat a particular case related to the example on the circle described in Section 5.1. Start from an IET $T$ on the circle with $n$ intervals of continuity, and cut the circle at a discontinuity point of $T$ that is not a discontinuity point of $T^{-1}$ (assuming that there is such a point). Then we get an IET $T_{0}$ on $[0,1)$. Looking at the interval of continuity of $T^{-1}$ that has been cut, we see that the underlying permutation $\tau \in \mathbb{S}_{n+1}$ of $T_{0}$ satisfies $\tau^{-1}(1)=\tau^{-1}(n+1)+1$. In other words, $\tau\left(i_{0}\right)=n+1$ and $\tau\left(i_{0}+1\right)=1$ for some $i_{0} \in\{1, \ldots, n\}$. Then define $T_{\theta} \in \mathrm{IET}_{\tau}$ to be the transformation with the same underlying permutation and the same lengths of continuity intervals $l_{i}^{\prime}=l_{i}$ for $i \neq i_{0}, i_{0}+1$, whereas $l_{i_{0}}^{\prime}=l_{i_{0}}-\theta$ and $l_{i_{0}+1}^{\prime}=l_{i_{0}+1}-\theta$ for some $\theta$ small enough. Denote by $t_{i}$ (resp. $t_{i}^{\prime}$ ) the translation lengths of $T$ (resp. $T_{\theta}$ ). Then one easily checks that $t_{i}^{\prime}=t_{i}+\theta$ for all $i=1, \ldots, n+1$ (we leave the proof of this fact to the reader). This shows that $\tau$ is driftable.

Proof of Proposition 5.12. Recall that the map $\Phi_{\sigma}$ is the map assigning translation lengths to interval lengths defined before Definition 5.7. Assume that $\sigma$ is not admissible, and consider $m$ such that $\sigma(m)=m$ and $\{1, \ldots, m-1\}$ is $\sigma$-invariant. Clearly, whatever the lengths of the continuity intervals, the corresponding interval exchange $T$ is the identity on $I_{m}$ (this follows from Lemma 5.4). This implies that for all $\overrightarrow{d l}=\left(d l_{i}\right)_{i \leq n}$ with $\sum d l_{i}=0$ the corresponding coordinate of $\Phi_{\sigma}(\overrightarrow{d l})$ vanishes. 
Now let us assume that $\sigma$ is admissible. Say that a pair $\left\{i_{1}, i_{2}\right\}$ is inverted if $i_{1}<i_{2}$ and $\sigma\left(i_{1}\right)>\sigma\left(i_{2}\right)$. The fact that $\sigma$ is admissible implies that each $i \in\{1, \ldots, n\}$ lies in an inverted pair since otherwise $\{1, \ldots, i-1\}$ and $\{i+1, \ldots, n\}$ are $\sigma$ invariant. Given an inverted pair $\left\{i_{1}, i_{2}\right\}$, consider the vector $\overrightarrow{d l}=\left(d l_{1}, \ldots, d l_{n}\right)$ where $d l_{i_{1}}=-1, d l_{i_{2}}=+1$, and $d l_{i}=0$ otherwise. Consider $\overrightarrow{d r}=\Phi_{\sigma}(\overrightarrow{d l})$ and write $\overrightarrow{d r}=\left(t_{k}\right)_{k=1 \ldots n}$ with $t_{k}=-\sum_{j=1}^{k-1} d l_{j}+\sum_{j=1}^{\sigma(k)-1} d l_{\sigma^{-1}(j)}$. The contribution of the first sum is 1 for $i_{1}<k \leq i_{2}$ and 0 otherwise. The contribution of the second sum is 1 for $\sigma\left(i_{2}\right)<\sigma(k) \leq \sigma\left(i_{1}\right)$ and 0 otherwise. It follows that $t_{k} \geq 0$ for all $k$, and that $t_{i_{2}}$ and $t_{i_{1}}$ are at least 1 .

Since any $i \in\{1, \ldots, n\}$ lies in an inverted pair, adding all vectors $\overrightarrow{d l}$ corresponding to all inverted pairs yields a vector whose image under $\Phi_{\sigma}$ has only positive entries. This proves that $\sigma$ is driftable.

\section{No infinite Kazhdan groups in IET}

Using non-distortion of cyclic subgroups in IET, Novak has proved that non-uniform lattices in higher rank semi-simple Lie groups with finite center have finite image in IET [9]. The following applies to lattices (including cocompact ones) in Kazhdan semi-simple Lie groups.

Theorem 6.1. Let $G$ be a finitely generated Kazhdan group. Then any morphism from $G$ to IET has finite image.

Here we give an argument based on the fact that orbits have polynomial growth.

Lemma 6.2. Let $G<$ IET be a finitely generated group. Then every orbit of $G$ for its action on $[0,1)$ has polynomial growth.

Proof. Let $g_{1}, \ldots, g_{n} \in$ IET be a finite set generating $G$ as a semigroup. For each $i$, let $t_{i}^{j} \in[0,1),\left(1 \leq j \leq m_{i}\right)$ be the translation lengths of $g_{i}$. Set $M=m_{1}+\cdots+m_{n}$ the total number of translations involved. For $R \in \mathbb{N}$, let $B_{R} \subset G$ be the set of all elements of word-length at most $R$. For any $x \in[0,1)$, commutation of translations implies that

$$
\left|B_{R} \cdot x\right| \leq R^{M}
$$

Recall that a group $G$ is Kazhdan (or has Kazhdan property $(T)$ ) if any unitary representation of $G$ having almost fixed vectors has a fixed vector. Witte Morris pointed out to us the following result:

Lemma 6.3 (See for instance Theorem B in [14]). Let $G$ be a finitely generated Kazhdan group and let $G \curvearrowright X$ be an action on a set. If the orbit of some $x \in X$ is infinite, then it has exponential growth. 
This implies that all orbits of $G$ are finite. We conclude the proof of Theorem 6.1 by the following fact.

Lemma 6.4. Let $G<$ IET be a finitely generated group. Assume that every orbit of $G$ is finite. Then $G$ is finite.

Proof. We view work in $\operatorname{IET}(\mathbb{R} / \mathbb{Z})$. Let $S$ be a finite generating set of $G$. Let $D \subset \mathbb{R} / \mathbb{Z}$ be the union of all orbits of the discontinuity points of the elements of $S^{ \pm 1}$. The set $D$ is finite and we can assume $D \neq \emptyset$ since otherwise the result is clear. All generators are continuous on the $G$-invariant set $\mathbb{R} / \mathbb{Z} \backslash D$. It easily follows that on each connected component of $\mathbb{R} / \mathbb{Z} \backslash D$, every orbit has the same finite cardinality. It follows that $G$ is finite.

\section{Residual finiteness}

Let $S$ be a finite set. A group marked by $S$ is a finitely generated group with a quotient map $\mathbb{F}_{S} \rightarrow G$, where $\mathbb{F}_{S}$ is the free group over $S$.

For $R>0$, denote by $B_{R}$ the ball of radius $R$ centered at 1 in $\mathbb{F}_{s}$. A sequence of marked groups $\pi_{n}: \mathbb{F}_{S} \rightarrow G_{n}$ converges to a marked group $\pi: \mathbb{F}_{S} \rightarrow G$ if for all $R>0$, we have $\operatorname{ker}\left(\pi_{n}\right) \cap B_{R}=\operatorname{ker}(\pi) \cap B_{R}$ for all $n$ large enough.

The aim of this section is to obtain the following.

Theorem 7.1. Every finitely generated subgroup of IET is the limit of a sequence of finite groups in the space of marked groups.

In particular, every finitely presented subgroup of IET is residually finite.

The second statement immediately follows from the first since any finitely presented marked group $G$ has a neighborhood consisting of quotients of itself (i.e., for all $\pi^{\prime}: \mathbb{F}_{S} \rightarrow G^{\prime}$ close enough to $\pi: \mathbb{F}_{S} \rightarrow G, \pi^{\prime}=q \circ \pi$ for some $\left.q: G \rightarrow G^{\prime}\right)$. This is because if $R$ is the length of the longest defining relation of $G$, then all defining relations are in $\operatorname{ker}(\pi) \cap B_{R}$, hence those relations are in $\operatorname{ker}\left(\pi_{n}\right)$ for sufficiently large $n$, so that $G_{n}$ is a quotient of $G$.

In particular Thompson's groups cannot embed in IET.

Corollary 7.2. Thompson's groups $F$ (on the interval) and $T, V$ (on the circle and the Cantor set) are not subgroups of IET.

Proof. It is known that $F, T$ and $V$ are finitely presented (cf. [1]). The groups $T$ and $V$ are simple and infinite, therefore not residually finite, and do not embed in IET by Theorem 7.1.

The group $F$ is not simple, but any proper quotient is abelian [1]. Since $F$ is infinite and non-abelian, it is not residually finite: any commutator has to be mapped to the trivial element in a finite quotient. Therefore, $F$ is not a subgroup of IET. 
The following is also immediate from Theorem 7.1. We would like to thank Alexei Muranov for turning our attention to the idea of sofic groups. See [4] or [13] for definitions.

\section{Corollary 7.3. The group IET is sofic.}

In view of Theorem 7.1, this follows immediately from the fact that finite groups are sofic, limits of sofic groups are sofic, and that soficity for a group holds if and only if it holds for its finitely generated subgroups [2].

The idea of the proof of the theorem is to produce a finite group by replacing the coefficients (i.e., the lengths) of the interval exchanges considered by rational numbers, keeping the fact that a given finite collection of words represent the identity or not. Then we use the fact that the group generated by interval exchanges with rational coefficients is always finite. The control on trivial and non-trivial elements is based on the fact that any system of linear equations and inequations with rational coefficients that has a real solution, also has a rational solution.

Proposition 7.4 ([8], Cor. 3.1.17). If a linear system of equations and inequations with rational coefficients has a real solution, then it has a rational solution.

In this statement we allow equalities $f(x)=0$ and strict inequalities $f(x)>0$ for $f: \mathbb{R}^{N} \rightarrow \mathbb{R}$ an affine map with rational coefficients.

Proof of Theorem 7.1. Define a rational polyhedron in $\mathbb{R}^{N}$ as the set of solutions of finitely many equations and inequations of the form $f(x)=0$ or $f(x)>0$ where $f: \mathbb{R}^{N} \rightarrow \mathbb{R}$ is an affine map. A $P L$ subset of $\mathbb{R}^{N}$ is a finite union of rational polyhedra. If $C \subset \mathbb{R}^{N}$ is a PL subset, we say that $f: C \rightarrow \mathbb{R}^{M}$ is $P L$ if there is a partition of $C$ into finitely many rational polyhedra in restriction to which $f$ coincides with an affine map $\mathbb{R}^{N} \rightarrow \mathbb{R}^{M}$ (note that contrary to standard terminology, we do not require $f$ to be continuous). Obviously, composition and inverses of PL maps are PL, and the preimage of a PL subset by a PL map is a PL subset. The intersection of finitely many PL subsets is a PL subset. By Proposition 7.4, any non-empty rational polyhedron contains a rational point, so does any non-empty PL subset.

Note that if $C_{1} \subset \mathbb{R}^{N_{1}}$ and $C_{2} \subset \mathbb{R}^{N_{2}}$ are PL subsets, then $C_{1} \times C_{2} \subset \mathbb{R}^{N_{1}+N_{2}}$ is a PL subset. Moreover, there is a PL embedding of $C_{1} \sqcup C_{2}$ to a PL subset of $\mathbb{R}^{N_{1}} \times \mathbb{R}^{N_{2}} \times \mathbb{R}$ as $C_{1} \times\{0\} \times\{0\} \cup\{0\} \times C_{2} \times\{1\}$.

Since for each permutation $\sigma \in \mathcal{S}_{n}, \mathrm{IET}_{\sigma}$ is parametrised by an open simplex of dimension $n-1$, and embeds naturally in $\mathbb{R}^{n}$, the set IET $\leq n$ of interval exchange transformations of $[0,1)$ with at most $n$ points of discontinuity can be identified with a PL subset of some $\mathbb{R}^{N_{n}}$.

The following lemma is a key.

Lemma 7.5. The group law $\mathrm{IET}_{\leq n} \times \mathrm{IET}_{\leq m} \rightarrow \mathrm{IET}_{\leq n+m}$ is a PL map. 
Proof. It is clear that the map $S \mapsto S^{-1}$ is PL. It is enough to prove that the group law is PL on $\operatorname{IET}_{\sigma} \times \operatorname{IET}_{\tau}$. Consider $(S, T) \in \mathrm{IET}_{\sigma} \times \mathrm{IET}_{\tau}$. Recall that $\Delta(S)$ denotes its set of discontinuity points (which we view as an ordered tuple $b_{1}<$ $\cdots<b_{n}$. Clearly, $\Delta(S)$ and $T \Delta(T)$ are affine functions of $(S, T)$. Thus each possible combinatorics for $\Delta(S) \cup T \Delta(T)$ defines a rational polyhedron, and we can assume that one of these combinatorics is fixed. Denote by $K_{k}$ the set of connected components of $[0,1) \backslash \Delta(S) \cup T \Delta(T)$, and note that $S \circ T$ is continuous on the intervals $T^{-1}\left(K_{k}\right)$. The endpoints of $K_{k}$ are affine functions of $(S, T)$. Since the evaluation map $[0,1) \times \operatorname{IET}_{\leq m} \rightarrow[0,1)$ sending $(x, T)$ to $T(x)$ is PL, the endpoints of $T^{-1}\left(K_{k}\right)$ are PL (indeed affine) functions of $(S, T)$. The amplitude of translation of $S \circ T$ on $T^{-1}\left(K_{k}\right)$ is the sum of the amplitude of $T$ on $T^{-1}\left(K_{k}\right)$ and of the amplitude of $S$ on $K_{k}$. It easily follows that the group law is PL.

Now consider a subgroup $G \subset$ IET generated by $g_{1}, \ldots, g_{k} \in \mathrm{IET}_{\leq n}$. We see $G$ as a quotient of the free group $\mathbb{F}_{k}$ over $\left\{x_{1}, \ldots, x_{k}\right\}$, in which $x_{i}$ is mapped on $g_{i}$. Denote by $B_{R} \subset \mathbb{F}_{k}$ the set of words of length $\leq R$. We need to show that, for all $R>0$, there is a finite quotient $Q$ of $\mathbb{F}_{k}$ such that for any element $w\left(x_{1}, \ldots, x_{k}\right) \in B_{R}, w\left(g_{1}, \ldots, g_{k}\right)$ is trivial in $G$ if and only if the image of $w$ in $Q$ is trivial.

For each $w \in B_{R}$, consider the PL map $f_{w}:\left(\mathrm{IET}_{\leq n}\right)^{k} \rightarrow \mathrm{IET}_{\leq R n}$ defined by $\left(t_{1}, \ldots, t_{k}\right) \mapsto w\left(t_{1}, \ldots, t_{k}\right)$. Consider $C_{w} \subset\left(\mathrm{IET}_{\leq n}\right)^{k}$ the PL subset defined by $C_{w}=f_{w}^{-1}(\{\mathrm{id}\})$ if $w\left(g_{1}, \ldots, g_{k}\right)=$ id in $G$ and by $C_{w}=f_{w}^{-1}\left(\mathrm{IET}_{\leq R n} \backslash\{\mathrm{id}\}\right)$ otherwise. The intersection $C$ of all $C_{w}$ is a PL subset that contains $\left(g_{1}, \ldots, g_{k}\right)$ by construction. It follows that $C$ contains a point with rational coordinates $\left(g_{1}^{\prime}, \ldots, g_{k}^{\prime}\right)$. In particular, the subgroup $Q$ of IET generated by $g_{1}^{\prime}, \ldots, g_{k}^{\prime}$ is finite. Because $\left(g_{1}^{\prime}, \ldots, g_{k}^{\prime}\right) \in C$, this finite quotient of $\mathbb{F}_{k}$ satisfies that $w\left(g_{1}, \ldots, g_{k}\right)$ is trivial in $G$ for all $w \in B_{R}$ if and only if $w\left(g_{1}^{\prime}, \ldots, g_{k}^{\prime}\right)$ is trivial in $Q$.

\section{An example of a subgroup}

Theorem 8.1. There is a subgroup $F \subset$ IET generated by two elements that contains

- an isomorphic copy of all finite groups,

- a free semigroup.

In particular this group is not linear and has exponential growth.

Proof. Let $\mathcal{C}=\mathbb{R} / 2 \mathbb{Z}$ be a circle of perimeter 2 and $\mathcal{I}=[0,1) \subset \mathcal{C}$ an interval of length 1 . Let $\mathcal{I}=[0,1)$ be a copy of $\mathcal{I}$. Consider the domain $\mathscr{D}=\mathscr{C} \cup \mathcal{I}$ and two iets $r, s$ on $\mathscr{D}$ such that $r$ is an irrational rotation on $\mathcal{C}$ and the identity on $\mathcal{L}$, and $s$ is the involution that switches $I$ and $J$ and is the identity on $C \backslash I$. 

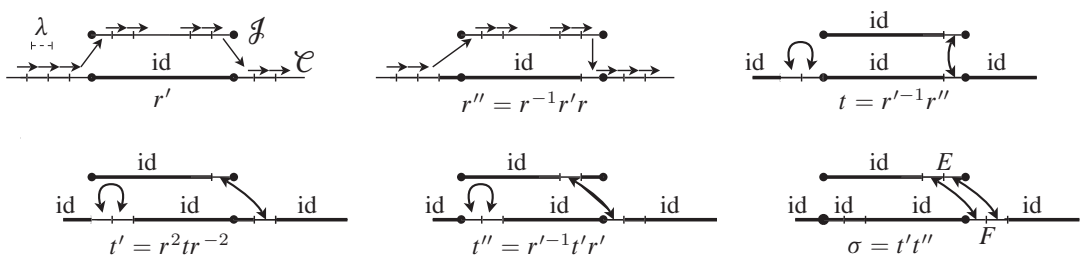

Figure 2. A non-linear example.

We prove that the subgroup $F=\langle r, s\rangle<$ IET contains every permutation group $\Sigma_{n}$. First, up to replacing $r$ with some power, we may assume that the amplitude $\lambda$ of $r$ satisfies $0<\lambda<\frac{1}{10 n}$.

We claim that $\langle r, s\rangle$ contains an involution $\sigma$ that exchanges $E=[1-2 \lambda, 1) \subset \mathcal{g}$ and $F=[1,1+2 \lambda) \subset \mathcal{C}$, and that is the identity everywhere else. The element $r^{\prime}=s r s$ can be thought as a rotation of the circle $\ell^{\prime}$ obtained from $\ell$ by replacing $\mathcal{I}$ by $\mathcal{G}$. Figure 2 shows the dynamics of $r^{\prime}, r^{\prime \prime}=r^{-1} r^{\prime} r, t=r^{-1} r^{\prime \prime}, t^{\prime}=r^{2} t r^{-2}$, $t^{\prime \prime}=r^{\prime-1} t^{\prime} r$, and finally $\sigma=t^{\prime} t^{\prime \prime}$.

Note that $r^{2}$ fixes $E$ and moves $F$ apart from itself. Since $\lambda \ll \frac{1}{n}$, the intervals $F$, $r^{2}(F), \ldots, r^{2 n}(F)$ are disjoint. It follows that the group $\left\langle\sigma, r^{2} \sigma r^{-2}, \ldots, r^{2 n} \sigma r^{-2 n}\right\rangle$ permutes the intervals $E, F, \ldots, r^{2 n}(F)$ and is isomorphic to $\Sigma_{n+2}$.

We now prove that the semigroup of $F$ generated by $r$ and $r^{\prime}=s r s$ is a free

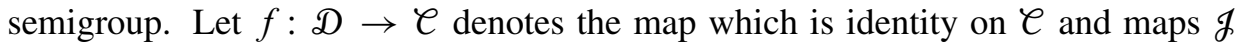
to $\mathcal{I}$ isometrically, and let $p, q$ be the initial and terminal endpoints of $\mathcal{I}$. Given a positive word $w$ on $\left\{r, r^{\prime}\right\}$, denote by $|w|$ its word length and by $W$ the corresponding transformation. Observe that for any $x \in \mathcal{D}$, there exists $n \in\{0, \ldots,|w|\}$ such that $f(W(x))=r^{n}(f(x))$. If $w \neq 1$, then for $x \in \mathcal{C} \backslash \mathcal{I}$ one has $n>0$, and since $r$ is irrational, this already implies $W \neq$ id. We note for future use that a similar argument shows that $W(p)=p$ if and only if $W$ is a power of $r^{\prime}$. We still have to check that the mapping $w \mapsto W$ is injective.

Define

$$
D_{k}=f^{-1}\left(\{p, q\} \cup r^{-1}\{p, q\} \cup \cdots \cup r^{-k+1}\{p, q\}\right)
$$

and

$$
D_{k}^{\prime}=f^{-1}\left(\{p, q\} \cup r\{p, q\} \cup \cdots \cup r^{k-1}\{p, q\}\right) .
$$

We see that for any positive word $w$, the discontinuity points of $W$ (resp. $W^{-1}$ ) are in $\left.D\right|_{w \mid}$ (resp. $\left.\left.D^{\prime}\right|_{w \mid}\right)$. Since $r$ is irrational, $p \notin r^{-1}\left(D_{k}\right)$, so $W r$ is continuous at $p$. We claim that $W^{\prime}=W r^{\prime}$ is discontinuous at $p$. Indeed, if $W^{\prime}$ was continuous at $p$, then since $r^{\prime}=W^{-1} W^{\prime}$ is discontinuous at $p, W^{-1}$ would be discontinuous at $W^{\prime}(p)$, so $\left.W^{\prime}(p) \in D^{\prime}\right|_{w \mid}$. Since $r$ is irrational and $p, q$ are not in the same orbit under $r$, this leaves only the possibility $W^{\prime}(p)=p\left(W^{\prime}(p)=s(p)\right.$ is impossible). It follows that $W^{\prime}$ is a power of $r^{\prime}$. Since $p$ and $q$ are not in the same orbit under $r$, 
no proper power of $r^{\prime}$ is continuous at $p$, so $W^{\prime}=\mathrm{id}$, a contradiction, which proves the claim.

We show that $W_{1}=W_{2}$ implies $w_{1}=w_{2}$ by induction on $n=\min \left(\left|w_{1}\right|,\left|w_{2}\right|\right)$. For $n=0$, this is the fact that $w \neq 1 \Rightarrow W \neq 1$. For $n>0$, the continuity or discontinuity of $W_{1}=W_{2}$ at $p$ tells us that $w_{1}$ and $w_{2}$ start with the same letter and, by induction, $w_{1}=w_{2}$. This concludes the proof that $r, r^{\prime}$ generate a free semigroup in IET.

Finally, the fact that $F$ is not linear follows immediately from the following fact.

Fact. Let $G$ be a finitely generated linear group. Then there exists $C$ such that the order of an element in $G$ of finite order is at most $C$.

This fact is proved in the proof of Theorem 36.2 (Schur) in [3], which says that any finitely generated periodic subgroup of $\operatorname{GL}(n, K)$ is finite if the characteristic of the field $K$ is zero. In the proof it is shown that if $G<\operatorname{GL}(n, K)$ is a finitely generated group then there exists $m$ such that if $g \in G$ has finite order then $g^{m}=1$. The essential part of the argument is to show that a characteristic root of $g$ is the $m$-th root of unity. For this part, it does not matter if the characteristic of $K$ is zero or positive. If the characteristic of $K$ is zero, it immediately follows that $g^{m}=1$. If the characteristic $p$ is positive, then we use a Jordan normal form of $g$, write $g^{m}=I+N$ with $N$ nilpotent, and observe that $\left(g^{m}\right)^{p^{n}}=1$.

\section{References}

[1] J. W. Cannon, W. J. Floyd, and W. R. Parry, Introductory notes on Richard Thompson's groups. Enseign. Math. (2) 42 (1996), 215-256. Zbl 0880.20027 MR 1426438

[2] Y. Cornulier, A sofic group away from amenable groups. Math. Ann. 350 (2011), 269-275. Zbl 1247.20039 MR 2794910

[3] C. W. Curtis and I. Reiner, Representation theory of finite groups and associative algebras. AMS Chelsea Publishing, Providence, RI, 2006. Zbl 1093.20003 MR 2215618

[4] M. Gromov, Endomorphisms of symbolic algebraic varieties. J. Eur. Math. Soc. (JEMS) 1 (1999), 109-197. Zbl 0998.14001 MR 1694588

[5] A. Katok and B. Hasselblatt, Introduction to the modern theory of dynamical systems. Encyclopedia Math. Appl. 54, Cambridge University Press, Cambridge 1995. Zbl 0878.58020 MR 1326374

[6] M. Kuranishi, On everywhere dense imbedding of free groups in Lie groups. Nagoya Math. J. 2 (1951), 63-71. Zbl 0045.31003 MR 0041145

[7] R. C. Lyndon and P. E. Schupp, Combinatorial group theory. Ergeb. Math. Grenzgeb. 89, Springer, Berlin 1977. Zbl 0368.20023 MR 0577064

[8] D. Marker, Model theory. Graduate Texts in Math. 217, Springer, New York 2002. Zbl 1003.03034 MR 1924282 
[9] C. F. Novak, Discontinuity-growth of interval-exchange maps. J. Mod. Dyn. 3 (2009), 379-405. Zbl 1183.37077 MR 2538474

[10] C. F. Novak, Continuous interval exchange actions. Algebr. Geom. Topol. 10 (2010), 1609-1625. Zbl 1203.37071 MR 2683746

[11] C. F. Novak, Interval exchanges that do not occur in free groups. Groups Geom. Dyn. 6 (2012), 755-763. Zbl 1268.37044 MR 2996410

[12] A. L. Onishchik and È. B. Vinberg, Foundations of Lie theory. In Lie groups and Lie algebras I, Encyclopaedia Math. Sci. 20, Springer, Berlin 1993, 1-94. Zbl 0781.22003 MR 1306738

[13] V. G. Pestov, Hyperlinear and sofic groups: a brief guide. Bull. Symbolic Logic 14 (2008), 449-480. Zbl 1206.20048 MR 2460675

[14] G. Stuck, Growth of homogeneous spaces, density of discrete subgroups and Kazhdan's property (T). Invent. Math. 109 (1992), 505-517. Zbl 0787.22011 MR 1176201

Received February 22, 2011; revised December 7, 2011

F. Dahmani, Institut Fourier - UMR 5582, Université de Grenoble, BP74, 38402 Saint Martin d'Hères cedex, France

E-mail: francois.dahmani@ujf-grenoble.fr

K. Fujiwara, Department of Mathematics, Kyoto University, Kyoto, 606-8502, Japan

E-mail: kfujiwara@math.kyoto-u.ac.jp

V. Guirardel, Université de Rennes 1, IRMAR, CNRS UMR 6625, 263 avenue du Général Leclerc, 35042 Rennes cedex, France

E-mail: vincent.guirardel@univ-rennes1.fr 